\title{
A Novel Image-Based Inertial Impact Test (IBII) for the Transverse Properties of Composites at High Strain Rates
}

\author{
L. Fletcher $^{1}$ (i) $\cdot$ J. Van-Blitterswyk ${ }^{1} \cdot$ F. Pierron ${ }^{1}$
}

Received: 16 June 2018 / Accepted: 23 January 2019 / Published online: 6 February 2019

(c) The Author(s) 2019

\begin{abstract}
Current methods for testing the high strain rate properties of composites require multiple assumptions that limit achievable strain rates. Therefore, this study presents a new method for testing the transverse properties of composites at high strain rates using ultra-high speed imaging. The image-based inertial impact test developed here uses the reflection of a compressive stress wave to generate tensile stress in the specimen. Throughout the test, full-field displacement measurements are taken. The acceleration and strain fields are then derived from the displacement fields. The acceleration is then used to calculate the average stress in the specimen. This paper describes the optimisation of the experimental configuration using simulations and the experimental validation of the technique. The elastic modulus and tensile strength were identified at strain rates of $\sim 2000$ $\mathrm{s}^{-1}$. The results showed an increase of $8 \%$ in elastic modulus and an increase of $57 \%$ in strength compared to quasi-static values.
\end{abstract}

Keywords Composites $\cdot$ High strain rate $\cdot$ Full-field measurement $\cdot$ Tensile strength $\cdot$ Virtual fields method

\section{Introduction}

In many applications, composite materials undergo high strain rate dynamic loading, for example: crash, impact and blast loading. For these cases, it is essential that the strain rate dependent material properties are accounted for in the design of composite structures. Computational power has exponentially increased in recent decades, allowing for extensive simulations of damage formation in composite structures. However, the usefulness of these simulations is dependent on the quality of the input constitutive models. The computational prediction of damage formed from impact loading in composites requires a full understanding of the complete failure envelope at high strain rates. This

Dr. Fletcher and Prof. Pierron are members of the Society of Experimental Mechanics.

L. Fletcher

1.c.fletcher@soton.ac.uk

J. Van-Blitterswyk

j.van-blitterswyk@ soton.ac.uk

F. Pierron

f.pierron@soton.ac.uk

1 Engineering Sciences, University of Southampton, Southampton SO17 1BJ, Hampshire, UK understanding can only be provided by detailed and careful experimental studies that are are used to calibrate material models. Unfortunately, practical difficulties related to high strain rate testing have limited the availability of data for the transverse properties of composites at high strain rates.

The strain rate dependent behaviour for the transverse direction in composites has been investigated in several previous studies [1-5]. However, it is difficult to directly compare data from these studies due to differences in the composite materials being tested (e.g. different fibre/matrix materials or different fibre volume fractions). The general trends for unidirectional (UD) composites show that the transverse properties exhibit strain rate sensitive behaviour. Specifically, the tensile elastic modulus shows a small increase or no change at high strain rates whereas the transverse tensile strength significantly increases with strain rate [1-4]. This behaviour is expected as the transverse properties are dominated by that of the matrix material. In general, the matrix is a polymeric material (e.g. epoxy) that shows relatively strong strain rate dependence $[6,7]$.

Current strength data for unidirectional composites at strain rates greater than $100 \mathrm{~s}^{-1}$ has been produced using the Split Hopkinson Pressure Bar (SHPB) technique [1-3, 5]. The SHPB technique is nominally capable of reaching strain rates on the order of $1000 \mathrm{~s}^{-1}$ provided that a number of assumptions are met. Two key assumptions of the SHPB 
analysis are: 1) quasi-static stress equilibrium and 2) one dimensional wave propagation (i.e. no dispersion effects). Violation of either of these assumptions leads to unreliable test results. The quasi-static equilibrium assumption is equivalent to neglecting inertial acceleration effects in the specimen. Once the quasi-static stress equilibrium condition is satisfied, one dimensional wave theory can be used to infer the material properties from strain gauge measurements on the bars of the SHPB apparatus. In order for this assumption to be satisfied, there are strict constraints on specimen size and geometry. Small specimens (relative to the overall bar length) are generally used such that the stress wave can reverberate multiple times and damp out quickly. Furthermore, the reverberation of the stress wave occurs during the initial (i.e. elastic) part of the test. Thus, it is generally accepted that in most cases it is not possible to accurately measure the elastic modulus using standard SHPB techniques and only an apparent modulus can be obtained [8,9]. Recently, it has been shown that for specific test configurations at moderate strain rates $\left(400 \mathrm{~s}^{-1}\right)$ inertial effects are probably small enough that the elastic modulus can be obtained with the standard SHPB analysis [10]. However, this finding is not generalisable to higher strain rates with lower wave speed materials such as the transverse composite samples considered here.

Achieving quasi-static stress equilibrium is particularly challenging when testing composite materials in transverse tension. The main reason for this is the brittle failure mode which is characterised by small strains to failure [1,2]. As the specimen fails at small strains, it can fail before inertial effects have damped out and the quasi-static stress equilibrium assumption will not be reasonably satisfied. The small strain to failure also means that the signal to noise ratio is low for measurement of the specimen strain using the gauges in the bars. The constraints of the quasi-static stress equilibrium assumption effectively limit the strain rate that can be obtained using the standard SHPB technique to under $1000 \mathrm{~s}^{-1}$ for the transverse tensile properties of a unidirectional composite $[1,2]$. The limitations of the standard SHPB technique for transverse properties of composites are shown clearly in a study by Melin and Asp [1]. In this study, Melin and Asp used a moiré fringe technique to analyse the strain field and compared this to the strain predicted by the standard SHPB technique. For this case, it was found that the SHPB theory underestimated the true strain field in the tested specimens. Additionally, in the study by Melin and Asp, it was only possible to measure the transverse properties up to an effective strain rate of $800 \mathrm{~s}^{-1}$. This limitation is due to the quasi-static stress equilibrium assumption of the SHPB technique as mentioned previously.

The second key requirement of the SHPB testing is the need for the stress wave imparted on the specimen to be onedimensional. Violation of this assumption is termed dispersion. The effects of dispersion generally manifest as oscillatory behaviour on the stress-strain response. This oscillatory behaviour is shown by the transverse stress-strain curve of figure 8 in ref. [2] at a nominal strain rate of $400 \mathrm{~s}^{-1}$. Pulse shaping techniques may be used to reduce the effects of dispersion by removing the high frequency content from the input pulse. However, this reduces the achievable strain rate. In any case, the effects of dispersion are never fully removed by pulse shaping [8]. To address the significant limitations for testing the transverse properties of composites at high strain rates, new test methods are required that do not rely on the assumptions of the standard SHPB technique.

Recent studies have shown that image-based full-field measurement techniques can provide a viable alternative for developing new high strain rate methods [11-13]. Full-field measurements provide a rich data set and allow for a multitude of measurement points across a test specimen. Techniques such as digital image correlation (DIC) and the grid method have been used extensively for quasi-static testing. When these techniques are combined with an inverse identification technique (e.g. the virtual fields method (VFM)) they can provide data to calibrate entire material models from a single test $[14,15]$. These techniques are now being applied to high strain rate testing due to advances in high speed imaging technology [11-13, 16, 17]. The recent advent of ultra-high speed cameras (as defined in ref. [18]) with framing rates greater than $1 \mathrm{MHz}$ has allowed for the imaging of stress wave propagation in materials. The inertial acceleration fields during stress wave propagation can be used to reconstruct stress information. In this way, the acceleration fields are used as an embedded load-cell in the specimen. This concept has been successfully demonstrated experimentally in several studies $[11-13,19]$. The use of ultra-high speed full-field measurements combined with the use of acceleration fields as an embedded load cell provides a promising new avenue for designing high strain rate tests for composite materials. Therefore, the overall aim of this study is to design a high strain rate test method for the transverse properties of composites using ultra-high speed full-field measurements. The following section of this paper describes the theory and concept design of the image-based inertial impact (IBII) test configuration. The next section of the paper then describes the finite element modelling used to validate the proposed test and optimise the test configuration. Following the finite element test design, the experimental campaign is discussed. The final sections of this paper outline the limitations and future applications of the new method along with a summary of the main findings.

\section{Concept Design and Theory}

\section{Imaged-Based Inertial Impact (IBII) Test Concept}

The aim of this work is to design a dynamic test to determine both the transverse stiffness and strength of a unidirectional 
composite. For this purpose, an image-based inertial impact (IBII) test has been designed similar to the test presented in Pierron and Forquin [13] for measuring the tensile properties of concrete. The basic principle behind this test is briefly described here. Consider the unidirectional composite specimen shown in Fig. 1, impacted by the force $F(t)$. A short compressive pulse is induced in the specimen. Then, the compressive pulse travels to the free edge of the specimen and reflects becoming tensile. Finally, the tensile wave travels through the specimen causing failure. Throughout the test, full-field displacement measurements are taken over the specimens' surface. From the full-field displacement data, the strain and acceleration fields can be calculated. The stiffness and strength of the material can then be determined from the kinematic fields using the methods described in the following sections. The indirect loading configuration is advantageous for testing brittle materials in tension as it takes away the need to grip the specimen and the associated difficulties with misalignment of the grips. Additionally, this configuration only requires that one edge of the sample is loaded with the other edges free with known boundary conditions. This simplifies the derivation of the relationships between the kinematic fields and the desired material properties as described in following sections.

In order to use the IBII test configuration, the compressive strength of the material must be higher than its tensile strength to avoid causing compressive failure with the initial pulse. The IBII test is well suited for testing the transverse properties of unidirectional composites as the compressive strength is much higher than the tensile strength. A unidirectional carbon fibre has a transverse compressive strength typically on the order of 200-300 MPa whereas the transverse tensile strength is in the range 40-80 MPa, depending on the matrix material. At high strain rates, both of these strength values are typically thought to increase $[1,2,4,5]$. Based on these properties, it is possible to select the compressive input

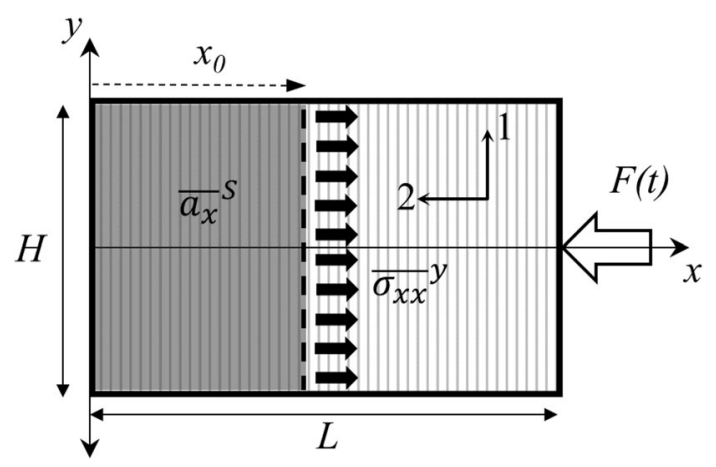

Fig. 1 Schematic of a unidirectional composite specimen impacted transverse to the fibre direction. Note that this is a free-body diagram of the test at an arbitrary time point $t$. The variables listed here illustrate those used in eq. 16 with the greyed out area showing the area over which the acceleration surface average is calculated pulse to cause tensile failure while remaining well below the compressive strength. It should be noted here that the transverse tensile strength at higher strain rates is thought to increase on the order of $30 \%[1,2]$. This projected increase in the tensile strength needs to be accounted for in the test design to ensure that the reflected tensile pulse causes failure in the specimen.

\section{The Virtual Fields Method}

Consider the schematic of a dynamically impacted composite specimen shown in Fig. 1. The underlying problem to address here is to relate the measured kinematic fields to the material constitutive parameters. In order to do this, the principle of virtual work is used. This approach is referred to as the virtual fields method (VFM). The interested reader is referred to ref. [15] for a detailed discussion of the VFM formulation. It is noted here that the principle of virtual work is simply the integral form of the stress equilibrium equation. The principal of virtual work, in absence of volume forces, is given by:

$-\int_{V} \boldsymbol{\sigma}: \boldsymbol{\epsilon}^{*} d V+\int_{\delta V} \boldsymbol{T} \cdot \boldsymbol{u}^{*} d S=\int_{V} \rho \boldsymbol{a} \cdot \boldsymbol{u}^{*} d V$

where $V$ is the volume of the specimen and $\delta V$ is the boundary surface of the volume. $\boldsymbol{T}$ is the Cauchy stress vector acting on the boundary surface $\delta V$, and $\sigma$ is the Cauchy stress tensor. The virtual displacement field is given by $\boldsymbol{u}^{*}$ and the virtual strain tensor (derived from $\boldsymbol{u}^{*}$ ) is given by $\boldsymbol{\epsilon}^{*}$. The acceleration field is denoted as $\boldsymbol{a}$ and $\rho$ is the material density. The matrix and vector dot products are denoted as : and respectively. The first term of eq. 1 is referred to as the internal virtual work, the second term is the external virtual work and the third term is the acceleration virtual work. It should be noted that all mechanical fields in this equation are functions of time and space. In order to simplify the equations here and in the following section, the time and space variables as function notation have been omitted.

In the VFM, the principle of virtual work is used to generate integral equilibrium equations that relate stress components to external forces and accelerations. The only conditions are that the virtual displacement vectors are continuous and piecewise differentiable. It is worth noting that the standard textbook statement that $\boldsymbol{u}^{*}$ has to be kinematically admissible is not a mathematical requirement for the equation but a need for unknown reaction forces at imposed displacement boundaries not to appear in the equation when it is used to solve the direct problem (as in the finite element method). Here, $\boldsymbol{u}^{*}$ has to be 'VFM-admissible' so that no unwanted unknowns appear in the equation (like a distribution of pressure in the grips of a test machine when only a 
resultant from a load-cell is measured). It is noted here that the selected virtual fields must also be $C^{0}$ continuous.

Additionally, a number of simplifying assumptions are made: (1) the density, thickness and stiffness of the specimen do not vary in space; (2) the kinematic fields are uniform through the thickness; and (3) the specimen is in a state of plane stress. As can be seen on Fig. 1, the stress field will be predominantly longitudinal, so the VFM analysis can concentrate on the $\sigma_{x x}$ term. For this purpose, generic virtual fields that cancel out the contributions of $\sigma_{y y}$ and $\sigma_{x y}$ are defined as follows:

$\left\{\begin{array}{l}u_{x}^{*}=f(x) \\ u_{y}^{*}=0\end{array}\left\{\begin{array}{l}\varepsilon_{x x}^{*}=f^{\prime}(x) \\ \varepsilon_{y y}^{*}=0 \\ \varepsilon_{x y}^{*}=0\end{array}\right.\right.$

where function $f(x)$ is continuous and piecewise differentiable. Therefore, the VFM equation becomes:

$-\int_{S} \sigma_{x x} \varepsilon_{x x}^{*} d S+\int_{l} T_{x} f(x) d l=\rho \int_{S} a_{x} f(x) d S$

where $S$ is the surface of the specimen and $l$ is the boundary of the specimen.

\section{Constitutive Model and Assumptions}

At this stage, it is necessary to substitute the stress in eq. 3 using an appropriate constitutive model. The material being analysed here is a unidirectional carbon fibre composite. For this material, a linear elastic orthotropic constitutive law is appropriate. Only the shear response can exhibit some non-linearity but this test will not produce significant shear strain in the material axes, and eq. 3 has been constructed to remove the shear contribution.

The stress-strain relationship for the $\sigma_{x x}$ component can be written as:

$\sigma_{x x}=Q_{22} \varepsilon_{x x}+Q_{21} \varepsilon_{y y}$

where $Q_{22}$ is the plane-stress stiffness transverse to the fibre direction and $Q_{21}$ is the plane-stress stiffness associated with Poisson's effect. They are provided by:

$Q_{22}=\frac{E_{22}}{1-v_{12} v_{21}} ; Q_{21}=v_{12} Q_{22}$

where $E_{22}$ is the Young's modulus in the transverse direction and $v_{12}$ and $v_{21}$ are the major and minor Poisson's ratios respectively. Additionally, the Young's modulus in the fibre direction $E_{11}$ is related to the transverse modulus $E_{22}$ as follows: $v_{21} E_{11}=v_{12} E_{22}$. Typical values of these parameters for a unidirectional carbon fibre composite are: $E_{11}=135 \mathrm{GPa}$, $E_{22}=10 \mathrm{GPa}$ and $v_{12}=0.3$, so $v_{21}=0.022$. Factorizing $Q_{22}$ in eq. 4 , the following equation is obtained: $\sigma_{x x}=Q_{22}\left(\varepsilon_{x x}+v_{12} \varepsilon_{y y}\right)$

Most of the $\varepsilon_{y y}$ in the test will arise from Poisson's effect because of the $\sigma_{x x}$ stress. Separating $\varepsilon_{y y}$ into the part arising from Poisson's effect denoted $\varepsilon_{y y}^{(P)}$ and the part caused by some possible presence of $\sigma_{y y}$ stress, denoted $\varepsilon_{y y}^{\left(\sigma_{y y}\right)}$, eq. 4 becomes:

$\sigma_{x x}=Q_{22}\left(\varepsilon_{x x}+v_{12}\left(\varepsilon_{y y}^{(P)}+\varepsilon_{y y}^{\left(\sigma_{y y}\right)}\right)\right)$

Now, we can replace $\varepsilon_{y y}^{(P)}$ by $-v_{21} \varepsilon_{x x}$ and factorize $\varepsilon_{x x}$. The term $1-v_{12} v_{21}$ simplifies with the denominator of $Q_{22}$ in eq. 5 and 7 becomes:

$\sigma_{x x}=E_{22} \varepsilon_{x x}+Q_{21} \varepsilon_{y y}^{\left(\sigma_{y y}\right)}$

Because of the high $E_{11}$ stiffness, $\varepsilon_{y y}^{\left(\sigma_{y y}\right)}$ will be very small and the second term in eq. 8 is negligible with respect to the first one. This will be checked in the finite element validation step later on this article, "Numerical Design and Validation" section. Therefore, in the rest of the article, the constitutive model that will be used is simply $\sigma_{x x}=E_{22} \varepsilon_{x x}$ and the VFM equation becomes (assuming that the material is homogeneous at the scale of the experiment):

$-E_{22} \int_{S} \varepsilon_{x x} \varepsilon_{x x}^{*} d S+\int_{l} T_{x} f(x) d l=\rho \int_{S} a_{x} f(x) d S$

\section{Virtual Fields For Stiffness Identification}

In the present work, several virtual fields have been used for different purposes. In the following section, a set of virtual fields are described that have been used for identifying the transverse elastic modulus $E_{22}$.

\section{Virtual Field 1}

Consider the simplest case, where $f(x)=1$ and $f^{\prime}(x)=0$. This virtual field describes a virtual rigid translation along the $x$ axis. Substituting this virtual field into eq. 9 removes the contribution of all virtual strain components. This leaves the external virtual work and acceleration virtual work terms, as follows:

$\int_{l} T_{x} d l=\rho \int_{S} a_{x} d S$

Equation 10 can be evaluated at any axial section along the specimen length, denoted $x_{0}$. This axial section is shown as a dashed line in Fig. 1. As the traction vector $\boldsymbol{T}=\boldsymbol{\sigma} \times \boldsymbol{n}, T_{x}$ is given by the stress component $\sigma_{x x}$. The resulting equation now becomes:

$\int_{-H / 2}^{H / 2} \sigma_{x x} d y=\rho \int_{-H / 2}^{H / 2} \int_{0}^{x_{0}} a_{x} d x d y$ 
In practice, the integral terms in eq. 11 are calculated using full-field displacement measurements obtained using the grid method or DIC. If these measurements have sufficient spatial resolution, the integrals can be approximated as discrete sums over a portion of the field of view. For example, the acceleration virtual work term can be approximated as follows:

$$
\begin{aligned}
& \int_{-H / 2}^{H / 2} \int_{0}^{x_{0}} a_{x} d x d y \approx \sum_{i=1}^{n} a_{x}^{(i)} s^{(i)} \\
& \frac{S}{n} \sum_{i=1}^{n} a_{x}^{(i)}=x_{0} H{\overline{a_{x}}}^{S}
\end{aligned}
$$

where $n$ is the total number of measurement points on the specimen surface bounded by the area $S\left[0 \leq x \leq x_{0}\right.$ $,-H / 2 \leq y \leq H / 2], a_{x}^{(i)}$ is the acceleration measured at point $i$ and $s^{(i)}$ is the small surface area associated with data point $i$. The small surface area $s^{(i)}$ is the same for all data points as the measurement grid is regular. ${\overline{a_{x}}}^{S}$ is the surface average of the acceleration over the field of view to the point $x_{0}$. The overline notation is used in subsequent equations to denote spatial averaging and a superscript $S$ denotes the spatial average over a portion of the field of view. The integral in the external virtual work term can be expressed in a similar manner:

$$
\begin{aligned}
& \int_{-H / 2}^{H / 2} \sigma_{x x} d y \approx \sum_{j=1}^{m} \sigma_{x x}^{(j)} s_{l}^{(j)} \\
& \frac{H}{m} \sum_{j=1}^{m} \sigma_{x x}^{(j)}=H{\overline{\sigma_{x x}}}^{y}
\end{aligned}
$$

where $m$ is the total number of points along the axial slice at $\left[x=x_{0},-H / 2 \leq y \leq H / 2\right], \sigma_{x x}^{(j)}$ is the axial stress at point $j$ and $s_{l}^{(j)}$ is the small section of the line associated with point $j$. The superscript $y$ coupled with the overline notation indicates the average over the line at $x_{0}$. The expressions in eqs. 13,15 can be substituted into eq. 11 to give:

${\overline{\sigma_{x x}}}^{y}=\rho x_{0}{\overline{a_{x}}}^{S}$

Equation 16 is hereafter referred to as the stress-gauge equation. The variables in eq. 16 are illustrated on the free body diagram in Fig. 1. The stress-gauge equation relates the mean axial stress to the mean of the acceleration field with the density acting as a calibration factor. Introducing the constitutive equation as defined previously, this equation becomes:

$E_{22}{\overline{\varepsilon_{x x}}}^{y}=\rho x_{0}{\overline{a_{x}}}^{S}$
By plotting $\rho x_{0} \bar{a}_{x}^{S}$ as a function of ${\overline{\varepsilon_{x x}}}^{y}$, a linear response should be obtained, the slope of which provides $E_{22}$.

\section{Virtual Field 2}

Additional virtual fields can also be used for stiffness identification. For instance, $f(x)=x-L$ and $f^{\prime}(x)=1$. This function has been selected such that $f(L)=0$. However, since $f(x)$ does not depend on $\mathrm{y}$, any virtual field as defined in eq. 2 would only involve the resultant stress at the impact edge, which can be obtained from eq. 16 when $x_{0}=L$, so for instance, $f(x)=x$ would lead to the linear combination of eqs. 16 and 18 below:

$\bar{\varepsilon}_{x x}^{S} E_{22}=-\rho \overline{a_{x}(x-L)} S$

for ${\overline{\varepsilon_{x x}}}^{S} \neq 0$, where $\overline{a_{x}(x-L)}$ is the average of the axial acceleration over the field of view weighted by the function $x-L$. Similarly, ${\overline{\varepsilon_{x x}}}^{S}$ is the average of the axial strain over the field of view. Using eq. 18 the transverse modulus can be determined at any frame of the dynamic test. The formula in eq. 18 is hereafter referred to as the manual VFM as it involves the manual definition of the function $f(x)$. Obviously, this raises the question regarding the choice of virtual field and how this affects the identified stiffness. In particular, using a virtual field that does not vary in time may lead to both terms in the fraction of eq. 18 to be zero and thus, to times when $E_{22}$ is unidentifiable. Moreover, for an ideal data set, with sufficiently rich acceleration and strain fields, the identified stiffness is insensitive to the choice of virtual field. However, in practice, the experimental acceleration and strain fields contain noise. Thus, the appropriate selection of virtual fields is dependent on minimising the effects of noise on the identification procedure. This problem has been previously investigated and methods have been developed to automatically select virtual fields that minimise the effects of noise [15, 20, 21]. This type of virtual field formulation is termed 'special optimised'. In the following section, the formalism of the special optimised virtual fields is developed for the case of identifying the transverse modulus of a unidirectional composite.

\section{Virtual Field 3: Special Optimised}

This follows the procedure presented in ref. [20]. Since the constitutive model is simplified here compared to the full orthotropic case in ref. [20], the main equations will be briefly recalled. Here, a set of piecewise functions have been used to expand the virtual fields.

To comply with the formulation in eq. 2 , the virtual mesh is formulated with only $u_{x}^{*}$ degree of freedom and nodes with the same $x$ coordinate must have the same $u^{*}$ value. If the number of nodes along the $x$ and $y$ axes are denoted by ' $n$ ' and ' $m$ ', 
respectively, then this gives a total of $m n$ conditions. The contribution of the impact load is also cancelled out by setting all $u^{*}=0$ for $x=L$ (impact edge), though this is not compulsory (see previous remark for virtual field 2). This results in an additional $m$ conditions. A typical virtual mesh is presented in Fig. 2.

The speciality condition is given by $\int_{S} \varepsilon_{x x} \varepsilon_{x x}^{*} d S=1$. This forces the virtual fields to 'follow' the deformation waves to produce non-zero internal and acceleration virtual works to ensure identification. This provides another constraining equation for the virtual fields. With this condition, eq. 9 becomes:

$E_{22}^{a p p}=-\rho \int_{S} a_{x} u^{*}(x) d S$

Here, $E_{22}^{a p p}$ is introduced to reflect the fact that the strain data is corrupted by noise. For exact data, $E_{22}^{a p p}=E_{22}$. If strain noise is present and is represented by a Gaussian distribution $\Pi_{x}$ of standard deviation $\gamma$, then eq. 9 becomes:

$E_{22}=\gamma E_{22} \int_{S} \Pi_{x} \varepsilon_{x x}^{*} d S-\int_{S} \rho a_{x} u_{x}^{*} d S$

It is further assumed that the noise amplitude is much smaller than the magnitude of the measured strains. Therefore, one can substitute the actual stiffness parameters with their approximate counterparts. This is to keep the process analytical. Therefore, eq. 20 can be rewritten as follows.

$E_{22} \simeq \gamma E_{22}^{a p p} \int_{S} \Pi_{x} \varepsilon_{x x}^{*(1)} d S+E_{22}^{a p p}$

Following through the same noise minimisation procedure as detailed in the work by Avril et al. [20] and approximating the integral quantities by discrete sums, the variance associated with $E_{22}$ is approximated by eq. 22 .

$\begin{aligned} V\left(E_{22}\right) & \simeq \gamma^{2}\left(\frac{S}{N}\right)^{2}\left[\left(E_{22}^{a p p}\right)^{2} \sum_{i=1}^{N}\left(\varepsilon_{x x}^{*}\left(M_{i}\right)\right)^{2}\right] \\ & =\left(\frac{S}{N}\right)^{2} E_{22}^{a p p} \cdot \boldsymbol{G} \cdot Q_{22}^{a p p}\end{aligned}$

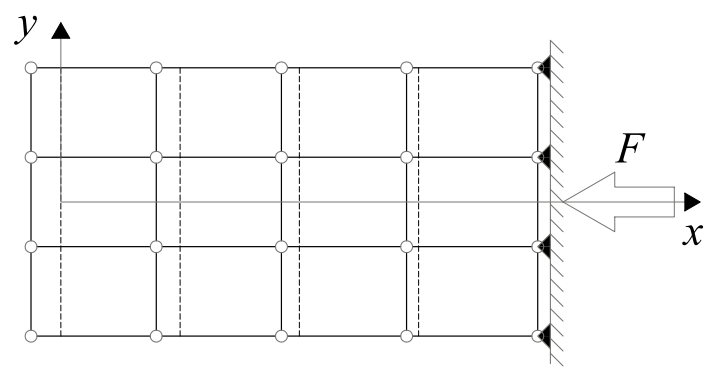

Fig. 2 Virtual mesh for the identification of $E_{22}$. Undeformed mesh shown in dashed lines. Only longitudinal virtual DOFs are allowed where $S$ is the surface area of the sample which has $N$ discrete measurement points. $M_{i}$ denotes the $i$ th measurement point and $\varepsilon_{x x}^{*}\left(M_{i}\right)$ is the virtual strain evaluated at this point. Since only one parameter is identified, the matrix $\boldsymbol{G}$ in ref. [20] is reduced to eq. 23.

$\boldsymbol{G}=\sum_{i=1}^{N}\left(\varepsilon_{x x}^{*(i)}\left(M_{i}\right)\right)^{2}$

Solving the minimisation problem using the Lagrangian multipliers method [20] produces a single set of optimal virtual field coefficients, which are used to obtain $E_{22}$.

$E_{22}=-\rho \frac{L H}{N}\left[\sum_{i=1}^{N} a_{x} \boldsymbol{Y}^{*} \cdot \tilde{u}_{x}^{*}\right]$

In eq. 24 the vector denoted $\boldsymbol{Y}^{(i) *}$ contains the unknown coefficients of the virtual fields, or $\tilde{u}_{x}^{*}$.

\section{Virtual Fields for Strength Identification}

It is also possible to use virtual fields to derive simple stress relationships from acceleration that can be used to approximate the stress state in a test. This can then be used to infer the failure stress in simple cases. Consider the stress-gauge formula given by eq. 16, this represents the mean axial stress in the specimen at a particular axial location. In this case, the stress is fully resolved in the $x$ direction (i.e. it provides the mean stress at each cross section). However, the distribution of the stress in the $y$ direction is only represented by an average value. Therefore, this will only be a reasonable approximation of the actual stress if the stress is uniform through the width. Only then will this equation be useful to identify strength.

Additional rigid body virtual fields can be used to derive measures of mean stress for strength measurement. This includes: (1) a virtual rigid body translation in the $y$ direction giving the mean shear stress ${\overline{\sigma_{x y}}}^{y}$ and (2) a virtual rigid body rotation giving the average of the first moment of the axial stress ${\overline{\sigma_{x x}}}^{y}$. These are derived in the following section. Consider the following virtual field that describes a rigid body translation in the $y$ direction:

$\left\{\begin{array}{l}u_{x}^{*}=0 \\ u_{y}^{*}=1\end{array}\left\{\begin{array}{l}\varepsilon_{x x}^{*}=0 \\ \varepsilon_{y y}^{*}=0 \\ \varepsilon_{x y}^{*}=0\end{array}\right.\right.$

Applying the same assumptions and reasoning as for the first rigid body virtual field, the principle of virtual work becomes:

$\int_{-H / 2}^{H / 2} \sigma_{x y} d y=\rho \int_{-H / 2}^{H / 2} \int_{0}^{x_{0}} a_{y} d x d y$ 
${\overline{\sigma_{x y}}}^{y}=\rho x_{0}{\overline{a_{y}}}^{S}$

where ${\overline{\sigma_{x y}}}^{y}$ is the average shear stress in the cross section at location $x_{0}$ and ${\overline{a_{y}}}_{y}^{S}$ is the surface average of the acceleration from the free edge to $x_{0}$. Next, consider the virtual field that describes a rigid body rotation about the origin:

$\left\{\begin{array}{l}u_{x}^{*}=y \\ u_{y}^{*}=-x\end{array}\left\{\begin{array}{l}\varepsilon_{x x}^{*}=0 \\ \varepsilon_{y y}^{*}=0 \\ \varepsilon_{x y}^{*}=0\end{array}\right.\right.$

With the same assumptions as before, the principle of virtual work becomes:

$$
\begin{aligned}
& \int_{-H / 2}^{H / 2} \sigma_{x x} y d y-x_{0} \int_{-H / 2}^{H / 2} \sigma_{x y} d y \\
& =\rho \int_{-H / 2}^{H / 2} \int_{0}^{x_{0}}\left(a_{x} y-a_{y} x\right) d x d y
\end{aligned}
$$

${\overline{\sigma_{x x} y}}^{y}-x_{0}{\overline{\sigma_{x y}}}^{y}=\rho x_{0}\left({\overline{a_{x} y}}^{S}-{\overline{a_{y} x}}^{S}\right)$

Substituting eq. 27 into eq. 30 gives the following equation for the average of the first moment of axial stress in terms of weighted averages of the acceleration field:

${\overline{\sigma_{x x} y}}^{y}=\rho x_{0}\left(x_{0}{\overline{a_{y}}}^{S}+{\overline{a_{x} y}}^{S}-{\overline{a_{y} x}}^{S}\right)$

where ${\overline{\sigma_{x x} y}}^{y}$ is the average of the first moment of the axial stress over the cross section at $x_{0}$. The acceleration terms in eq. 31 are spatial averages over the specimen surface up to the transverse slice $x_{0}$. Equations 16,27 and 31 can now be used to provide an $x$-resolved linear approximation of the $\sigma_{x x}$ stress along $y$. Denoting $\sigma_{x x}(L S G)$ the linear approximation of the $\sigma_{x x}$ stress along $y$ (LSG for 'Linear Stress-Gauge'). $\sigma_{x x}(L S G)$ can be expressed as follows:

$\sigma_{x x}(L S G)=\sigma_{x x}^{a}+\frac{y}{H} \sigma_{x x}^{b}$

where $\sigma_{x x}^{a}$ and $\sigma_{x x}^{b}$ are the coefficients of the linear function. Consider the first rigid body virtual field describing a translation in the $x$ direction. Equation 32 can be substituted into eq. 11 to obtain:

$\int_{-H / 2}^{H / 2}\left(\sigma_{x x}^{a}+\frac{y}{H} \sigma_{x x}^{b}\right) d y=\rho \int_{-H / 2}^{H / 2} \int_{0}^{x_{0}} a_{x} d x d y$

Evaluating the integrals and simplifying with the overline notation yields the coefficient $\sigma_{x x}^{a}$ :

$\sigma_{x x}^{a}=\rho x_{0}{\overline{a_{x}}}^{S}$ which is, not surprisingly, eq. 16. Next, consider the virtual field describing a rigid body rotation. Substituting eqs. 32 into 29 gives:

$$
\begin{aligned}
& \int_{-H / 2}^{H / 2}\left(\sigma_{x x}^{a}+\frac{y}{H} \sigma_{x x}^{b}\right) y d y-x_{0} \int_{-H / 2}^{H / 2} \sigma_{x y} d y \\
& =\rho \int_{-H / 2}^{H / 2} \int_{0}^{x_{0}}\left(a_{x} y-a_{y} x\right) d x d y
\end{aligned}
$$

Simplifying the integrals in eq. 35 yields:

$\sigma_{x x}^{b} \frac{H}{12}-x_{0}{\overline{\sigma_{x y}}}^{y}=\rho x_{0}\left({\overline{a_{x} y}}^{S}-{\overline{a_{y} x}}^{S}\right)$

The term ${\overline{\sigma_{x y}}}^{y}$ can be expressed in terms of acceleration averages using eq. 27 . If the relation in eq. 27 is substituted into eq. 36 , the coefficient $\sigma_{x x}^{b}$ can be expressed as:

$\sigma_{x x}^{b}=\frac{12 \rho x_{0}}{H}\left(\bar{a}_{x}{ }^{S}-{\overline{a_{y} x}}^{S}+x_{0}{\overline{a_{y}}}^{S}\right)$

Finally, a linear approximation of the stress distribution is obtained by combining the results for each of the linear coefficients with eq. 32 :

$\sigma_{x x}(L S G)=\rho x_{0}{\overline{a_{x}}}^{S}+\frac{12 \rho x_{0} y}{H^{2}}\left(\overline{a_{x} y} S-\overline{a_{y} x} S+x_{0}{\overline{a_{y}}}^{S}\right)$

The eq. 38 will be hereafter referred to as the linear stressgauge formula. This equation is useful as it allows for an improved approximation of the stress field in the $y$ direction compared to the standard stress-gauge formula in eq. 16 . A valid question that arises in the application of the linear stress-gauge formula is: "How accurate is the linear approximation compared to the true stress field?". This can be addressed by using the constitutive law identified with the previously described methods. The stress field in the specimen can be reconstructed from the strain using the identified constitutive law. This can then be compared to the stress predicted by the linear stress-gauge approach to assess the accuracy of the approximation. Obviously, once the assumptions of the constitutive law are violated then this comparison no longer holds. For instance, if the identified constitutive law is linear elastic then once the material exhibits nonlinear behaviour (plasticity, damage, failure etc.), these two measures of stress will diverge. In fact, this divergence can be useful in identifying the point at which a linear elastic material begins to damage and fail. This approach will be used in this study to identify the transverse tensile strength.

The VFM equations using rigid body virtual fields can all be used to approximate the stress state in a material at failure and therefore provide an estimate of the materials strength. Compared to other virtual fields, these equations provide a stress information which is resolved in the $x$ direction (i.e. averages over a cross-section) while other virtual 

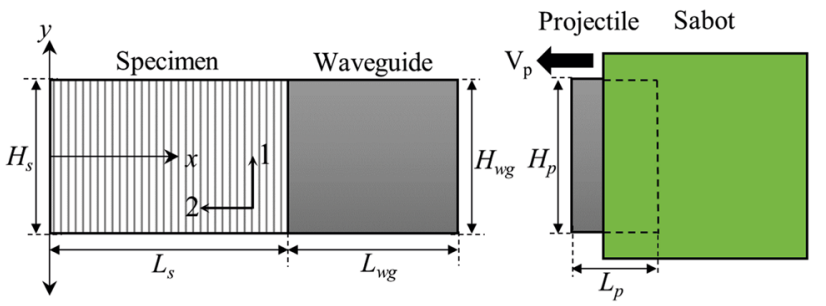

Fig. 3 Schematic of test configuration showing the main test components and relevant geometric variables. For the experiments and the finite element model the specimen is a thin rectangular plate and the remaining components are cylindrical. For the finite element modelling the sabot is neglected

fields provide some spatial average over some portion or all of the $(x, y)$ plane. Virtual fields that provide stress averages over a large planar area cannot be used for strength identification due to inadequate spatial resolution. It is worth noting however that the stress averages are not resolved in the $y$ direction. Only a first moment can be derived (see eq. 38) that can provide a linear approximation of the stress through the width. Whether the stress components could be reconstructed with better spatial resolution from acceleration is an open problem. Fundamentally, this is difficult to address as three stress components are required from only two acceleration components.

\section{Numerical Design and Validation}

The first step in designing a new test technique is to validate the proposed methodology using numerical simulation. Here, numerical modelling has two purposes: (1) select test parameters to maximise the reflected tensile stress while minimising the input compressive stress; and (2) validate the stiffness identification procedures described previously in "Concept Design and Theory" section.

\section{Model Configuration and Parametric Design Sweep}

In order to experimentally implement the impact test in Fig. 1, a number of components are required; these are shown in Fig. 3. To impart the initial compressive pulse into the rectangular specimen, a short cylindrical projectile is used. The projectile is encased in a nylon sabot to reduce friction with the gas gun barrel. In order to minimise effects of small misalignments between the projectile and specimen, a cylindrical waveguide is used between the specimen and the projectile. The waveguide acts like an end tab on typical quasi-static test specimen. Instead of mitigating the effects of the grips in a mechanical test machine the waveguide allows for three dimensional effects in the input stress pulse to be mitigated by the Saint Venant effect before
Table 1 Summary of parametric sweep variables for test design simulation

\begin{tabular}{llll}
\hline Parameter & Min & Max & Increment \\
\hline Projectile length, $L_{p}(\mathrm{~mm})$ & 10 & 50 & 5 \\
Projectile velocity, $V_{p}(\mathrm{~m} / \mathrm{s})$ & 5 & 50 & 5 \\
Waveguide length, $L_{w g}(\mathrm{~mm})$ & 40 & 60 & 10 \\
\hline
\end{tabular}

transmitting the loading pulse into the specimen [22]. The choice of geometry for the various components in Fig. 3 influences the ratio of input compressive stress to reflected tensile stress. In order to achieve the desired tensile stress and optimise the ratio of input compressive stress to tensile stress, a parametric design sweep is required. This design sweep was conducted using an explicit dynamics finite element model described in the following section. Before describing the finite element model, the variables in Fig. 3 will be considered with respect to experimental constraints.

Based on the available experimental apparatus, the geometry of the specimen and test components are constrained. This removes several variables from the parametric design sweep. The bore of the gas gun to be used for the impact experiments is limited to projectiles of $45 \mathrm{~mm}$ diameter (with a $50 \mathrm{~mm}$ diameter sabot). For simplicity, the projectile diameter, waveguide diameter and specimen height have been fixed at $45 \mathrm{~mm}\left(H_{s}=H_{w g}=H_{p}=45 \mathrm{~mm}\right)$. Further constraints on specimen geometry arise from the aspect ratio of the camera used for the full-field measurements. Specifically, to maximise the spatial resolution of the full-field displacement measurements, the aspect ratio of the specimen should match the aspect ratio of the camera. For the experiments performed in this study, a Shimadzu HPV-X camera was used with an aspect ratio of 1.6:1. Additionally, an allowance is required for rigid body motion of the sample during the test such that the free edge of sample does not move out of the field of view. Therefore, the sample length $L_{s}$ is fixed at $70 \mathrm{~mm}$ allowing for $2 \mathrm{~mm}$ of rigid body motion.

The remaining test parameters can be chosen based on a design sweep to optimise the test configuration. The remaining parameters to be selected include: projectile length $L_{p}$, projectile velocity $V_{p}$, and waveguide length $L_{w g}$. The ranges of these variables that were simulated are summarised in Table 1. Based on the composite material that will be used for the experimental studies, the transverse tensile strength was assumed to be $\sim 50 \mathrm{MPa}$. This was later found to be appropriate based on the quasi-static test campaign and includes an allowance for an increase with strain rate (see "Composite Material and Quasi-Static Tests" section). Data was not available for the transverse compressive strength of this specific material. Therefore, the compressive strength was conservatively estimated to be at least $200 \mathrm{MPa}$ based on the results in ref. [5, 23]. This gives a design envelope of 
${\overline{\sigma_{x x}}}^{y}>50 \mathrm{MPa}$ during tensile loading and ${\overline{\sigma_{x x}}}^{y}>-200 \mathrm{MPa}$ during the compressive loading. While it is possible to optimise the design based on the local stress rather than the average the average stress has been selected here as it will give a conservative prediction for the maximum tensile stress that is reached.

\section{Finite Element Implementation}

The explicit dynamics simulations for this study were conducted using ANSYS APDL LS-DYNA (v 16.2). A schematic of the test configuration is shown in Fig. 3. The parametric design sweep was conducted using a 3D finite element model. The specimen was meshed with quadrilateral SOLID164 elements (8-node, linear, reduced integration) and the cylindrical waveguide and projectile were meshed with tetrahedral SOLID168 elements (10-node, quadratic). The sabot shown in Fig. 3 was neglected in the 3D simulation for computational efficiency. Contact was modelled between all components using the default, automatically generated, three dimensional contact algorithm in LS-DYNA.

A convergence study was conducted using a parametric sweep of mesh size, time step and beta damping (stiffness proportional damping). Note that beta damping was used to reduce the numerical errors that occur as high frequency oscillations in the acceleration fields. The simulation parameters were selected from this sweep in order to minimise the error between the average stress ${\overline{\sigma_{x x}}}^{y}$ calculated from the accelerations (eq. 16) and the average stress extracted from the finite element model. The selected simulation parameters are summarised in Table 2. As the specimen behaviour was of greatest interest the mesh size of the projectile and waveguide was set to be more coarse than the specimen with an element edge length of $4 \mathrm{~mm}$ (compared to $0.5 \mathrm{~mm}$ elements for the specimen).

The boundaries of all components were assumed to be free. The only condition applied to the simulation was the initial velocity of the projectile. The total simulation time was set such that the stress wave generated by the impact between the projectile and waveguide could travel through the waveguide and the specimen to the free edge, reflect and travel back through the length of the specimen again. All materials were modelled as homogeneous and linear elastic. The unidirectional carbon fibre composite specimen was modelled as orthotropic with material properties $\rho=1600$ $\mathrm{kg} / \mathrm{m}^{3} E_{11}=135 \mathrm{GPa}, E_{22}=E_{33}=10 \mathrm{GPa}, v_{12}=v_{13}=0.02$ $v_{23}=0.3, G_{12}=G_{13}=5 \mathrm{GPa}$ and $G_{23}=3.85 \mathrm{GPa}$. The projectile and waveguide material were modelled as Aluminium 6061 -T6 with isotropic material properties $\rho=2700 \mathrm{~kg} / \mathrm{m}^{3}$, $E=70 \mathrm{GPa}$ and $v=0.33$. The initial distance between the projectile and the waveguide was set based on the projectile
Table 2 Explicit dynamics simulation parameters

\begin{tabular}{ll}
\hline Specimen mesh size & $0.5 \mathrm{~mm}$ \\
Nominal time step & $0.5 t_{\text {crit }}=0.1 \mu \mathrm{s}$ \\
Beta damping & 0.1 \\
Data output step & $0.5 \mu \mathrm{s}$ \\
\hline
\end{tabular}

velocity such that there were three output steps before the projectile contacted the waveguide.

\section{Parametric Sweep Results}

The parametric sweep results showed that the length of the waveguide $\left(L_{w g}\right)$ had minimal effect on the input compressive and reflected tensile stresses. This was the case as long as the waveguide was at least twice the projectile length. When the projectile is the shortest component, the projectile length controls the pulse duration (i.e. the pulse duration is twice the time it takes to traverse the length of the projectile). Therefore, as long as the waveguide is at least double the projectile length, it will not truncate the overall pulse duration. Based on practical considerations, the length of the waveguide was selected to be $50 \mathrm{~mm}$. This choice was made to minimise the mismatch in mass between waveguide and projectile to reduce the potential for the projectile to reflect back towards the barrel and cause potential damage to it.

The results of the parametric sweep for the remaining parameters are shown in Fig. 4. It desirable to maximise the proportion of input compressive stress that is reflected as tensile stress. Therefore, the ratio of reflected tensile stress to input compressive stress is shown in Fig. 5. It is interesting to note that in Fig. 5, the curves are effectively insensitive to the projectile velocity and collapse onto each other. The combination of Figs. 4 and 5 suggests that the projectile length determines the ratio of reflected tensile to compressive stress while the projectile velocity determines the magnitude of the input compressive stress.

From the data shown in Figs. 4 and 5, it is possible to select a wide range of parameters that will achieve the desired design constraints. The design envelope that satisfies the requirements is given by: projectile velocity $\left[20 \leq V_{p} \leq 40 \mathrm{~m} / \mathrm{s}\right]$ and projectile length $\left[10 \leq L_{p} \leq 50\right.$ $\mathrm{mm}]$. The configuration selected for the experiments is as follows: waveguide length of $L_{w g}=50 \mathrm{~mm}$, projectile length of $L_{p}=25 \mathrm{~mm}$ and projectile velocity of at least $V_{p}=30$ $\mathrm{m} / \mathrm{s}$. This configuration leads to a reflected average tensile stress of $105 \mathrm{MPa}$ and an input average compressive stress of $-188 \mathrm{MPa}$. It should be noted that the selected configuration is not the most efficient. The reason for this selection was based on economical reasoning; that is, the projectile design was suitable for testing several materials (e.g. soda-lime 
(a)
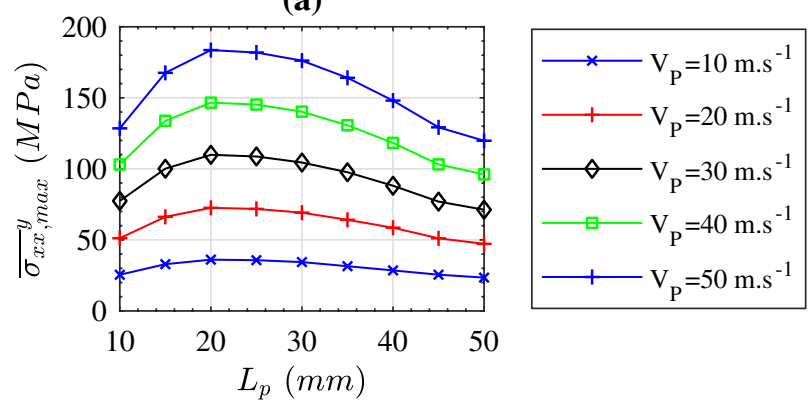

Fig. 4 Parametric sweep results for the 3D finite element model a the maximum average tensile stress ${\overline{\sigma_{x x} \text { max }}}^{y}$ reflected and $\mathbf{b}$ the maximum input compressive stress ${\overline{\sigma_{x x}, \min }}_{\text {in }}^{y}$ plotted against the projectile length $L_{p}$
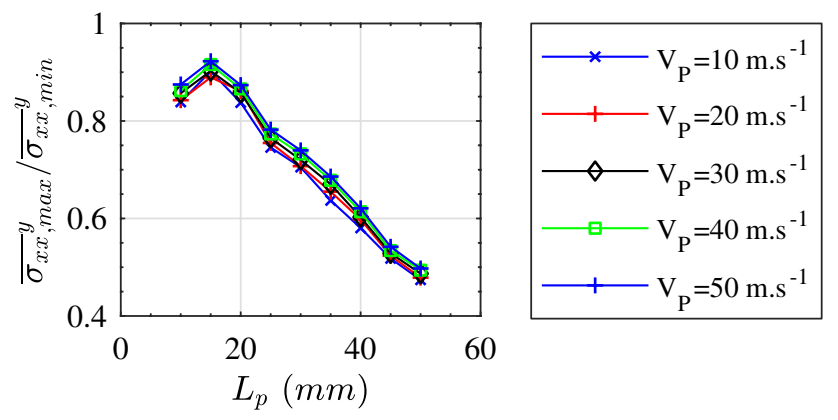

Fig. 5 Ratio of reflected average tensile stress to input compressive stress from the 3D finite element model ${\overline{\sigma_{x x}, \text { max }}}^{y} /{\overline{\sigma_{x x}, \min }}^{y}$. Note that every second velocity value has been plotted for clarity

glass) using an IBII test and allowed for a large batch of projectiles to be made, reducing costs.

For the selected test configuration the finite element model predicts that the maximum average tensile stress occurs at $x=11.25 \mathrm{~mm}$ and $t=42.5 \mu \mathrm{s}$. The $\sigma_{x x}$ stress field at $t=42.5 \mu$ is shown in Fig. 6a. The stress field in Fig. 6a is interesting as the peak stress occurs in the centre of the specimen. Therefore, it is possible that the specimen will fail in the centre rather than at the edges. The predicted maximum average tensile stress at different different axial locations is shown in Fig. 6b. Analysis of Fig. 6b shows that just after the the first tensile peak another occurs near the centre of the specimen length. If the first tensile peak does not cause failure it is possible that a failure will occur near the centre of the specimen length. If it is assumed that the failure strength of the tested material is at least $50 \mathrm{MPa}$ then the model predicts that failure will occur between $x=5$ to $40 \mathrm{~mm}$ and $t=41$ to $52 \mu \mathrm{s}$.

As the purpose of this parametric design sweep is to design a high strain rate test, it is also interesting to analyse the predicted strain rates that are achievable. In Fig. 7, the strain rate fields are shown for a time before the wave has reflected (a), and following the reflection of the wave (b)

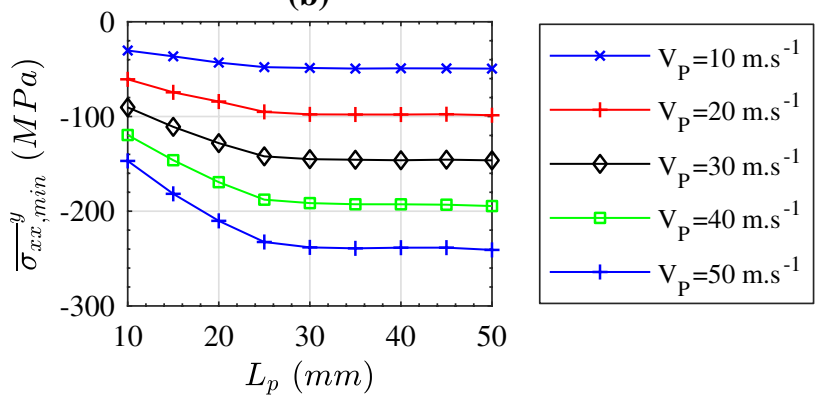

and projectile velocity $V_{p}$. The waveguide length $L_{W G}=50 \mathrm{~mm}$. Note that every second velocity value has been plotted for clarity
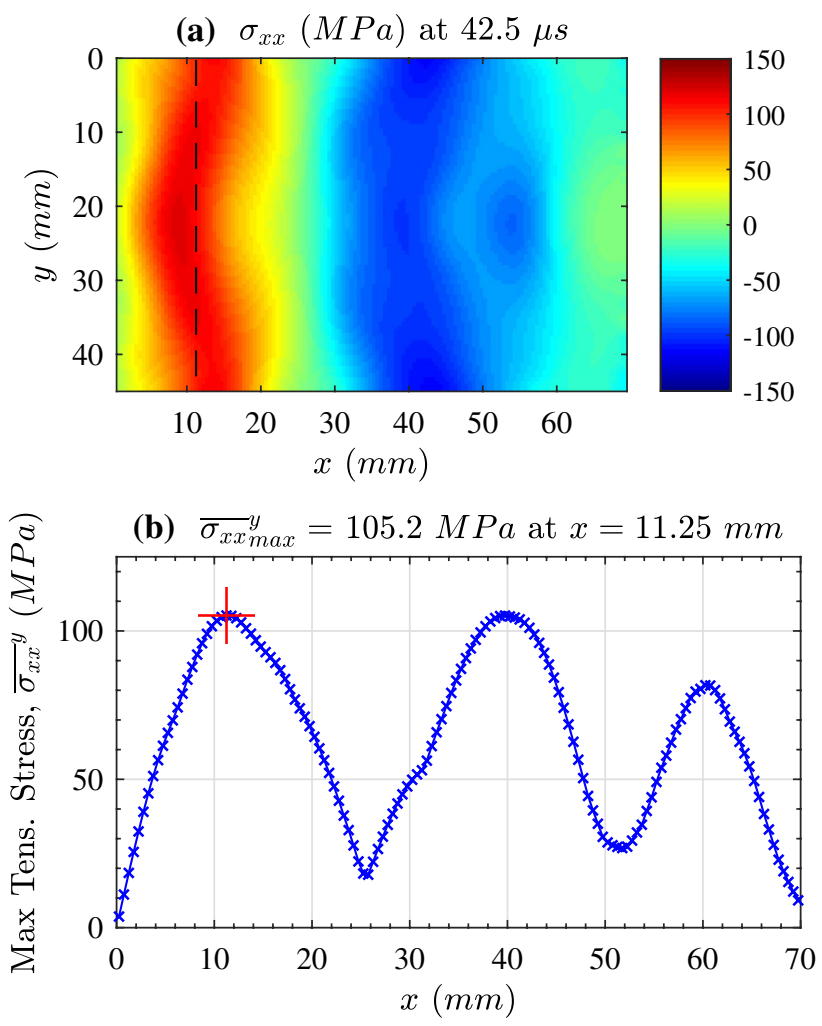

Fig. 6 a Finite element model (3D case) stress field $\sigma_{x x}$ for the selected test configuration indicating the time and location of maximum average tensile stress. b Maximum average tensile stress as a function of axial location. The predicted maximum average tensile stress is highlighted with a red cross. (Color figure online)

(b). These figures show that for the IBII test the strain rate field is heterogeneous over the specimen surface. Further measures of the axial strain rate can be obtained by averaging over the whole field of view as in Fig. $7 \mathrm{c}$ or averaging over the width at a given axial position as in Fig. 7 d. For the selected test configuration, the finite element model predicts peak width averaged strain rates on the order of $2000 \mathrm{~s}^{-1}$. 


\section{Stiffness Identification Validation}

Here a finite element model will be used to validate the stiffness identification methods proposed in "Virtual Fields For Stiffness Identification" section. In order to have sufficient mesh density for the acceleration and stress field to be in equilibrium it was necessary to run a $2 \mathrm{D}$ plane stress model that only considered the sample. The validity of using a 2D plane stress assumption for the specimen was assessed using the results of the 3D model as detailed in the Appendix. An additional convergence study was conducted on the finite element model for the stiffness identification procedures. The results of this study showed that the previously selected damping and time step parameters were suitable. However, a finer mesh of $0.25 \mathrm{~mm}$ was required. The sample was meshed using PLANE162 elements (4-node, reduced integration, plane stress) and the loading was applied using a pressure pulse applied to the impacted edge of sample. The magnitude and temporal evolution of the loading pulse was set to replicate the loading conditions observed in the 3D model. All other edges of the sample were considered to be free.

Full-field strain and acceleration maps were extracted from the finite model at each output time step. The acceleration and strain fields are shown in Fig. 8 at a time point when the compressive wave has fully entered the specimen. The acceleration fields from the finite element model were used to calculate the average stress ${\overline{\sigma_{x x}}}^{y}$ according to eq. 16 . Stress-strain curves where then plotted at all axial sections along the specimen length using the average axial strain ${\overline{\varepsilon_{x x}}}^{y}$. The stress-strain curves were fitted with a linear function to obtain the elastic modulus $E_{22}$. A stress-strain curve reconstructed near the specimen centre $(x=35 \mathrm{~mm})$ is shown in Fig. 9a. The identified
Fig. 7 Predicted strain rate from the 3D finite element model. Strain rate maps over the specimen surface at $t=24.5 \mu \mathrm{s}$ (a) and at $t=50 \mu \mathrm{s}$ (b). c Average strain rate over the specimen surface $\overline{\varepsilon_{x x}}$ for the test duration and $\mathbf{d}$ axial averaged strain rate ${\overline{\varepsilon_{x x}^{-}}}^{y}$ at $x=35 \mathrm{~mm}$. (Color figure online)
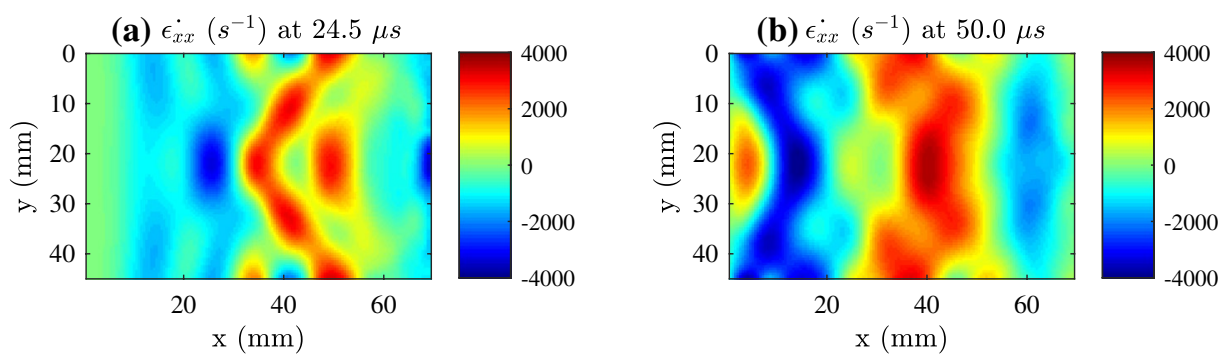

(c)
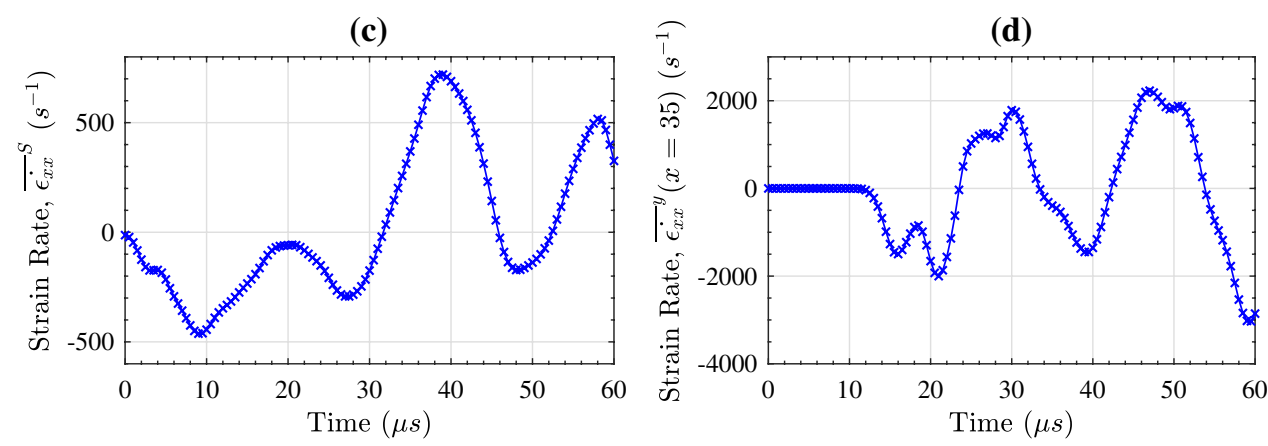

Fig. 8 Finite element model (2D case) strain and acceleration fields for the configuration: wave guide length $L_{w g}=50$ $\mathrm{mm}$, projectile length $L_{p}=25$ $\mathrm{mm}$ and projectile velocity $V_{p}=30 \mathrm{~m} / \mathrm{s}$ at $t=24.5 \mu \mathrm{s}$. (Color figure online)
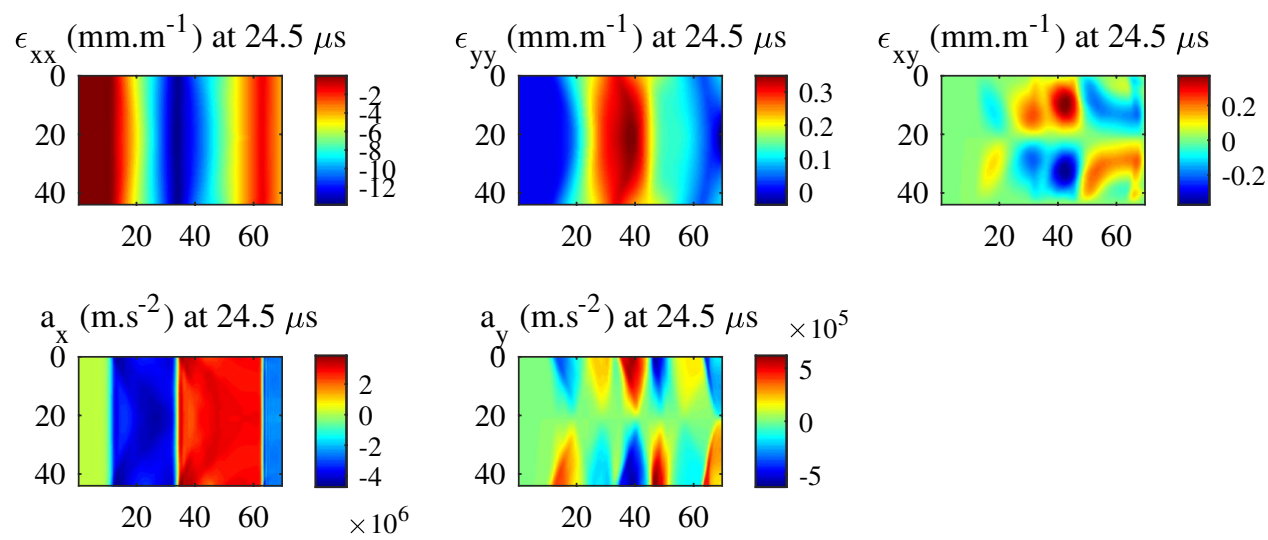
elastic modulus from linearly fitting all stress-strain curves along the specimen length is shown in Fig. 9b. Analysis of Fig. 9 shows that the identification from the finite element data is stable and within $2 \%$ of the input value over the specimen length.

The stiffness identification procedure was also validated for the manual and special optimised virtual fields using the finite element data. The identification using the manual virtual field procedure is shown in Fig. 10a and the identification using the special optimised virtual field is shown in Fig. 10b. For the special optimised virtual fields, a virtual mesh of 5 by 1 elements was used. A virtual mesh refinement study was conducted and showed that the identification converged with a virtual mesh of 3 by 1 elements. Here, 5 by 1 elements are used to be consistent with the convergence study performed on experimental data. For both types of virtual fields, the identification is of poor quality at the start of the test when the wave is entering the specimen. The reason for this is that there is inadequate spatial information to identify the material properties (i.e. strain and acceleration). The manual virtual fields show additional points of instability which correspond to to a situation where both terms in eq. 18 come close to zero. This instability is not evident in the special optimised virtual fields. The reason for this is that the special optimised virtual fields follow the input spatial information leading to an improved identification. This is shown in Fig. 11 where the $u_{x}^{*}$ special optimised virtual field highlights the acceleration peak.

When the identification is stable the variation is low and well under $1 \%$ of the input target value. All stiffness identification methods show excellent agreement with the input stiffness value when applied to finite element data. This validates the approach and the next section is dedicated to the experimental implementation of the method. (a)

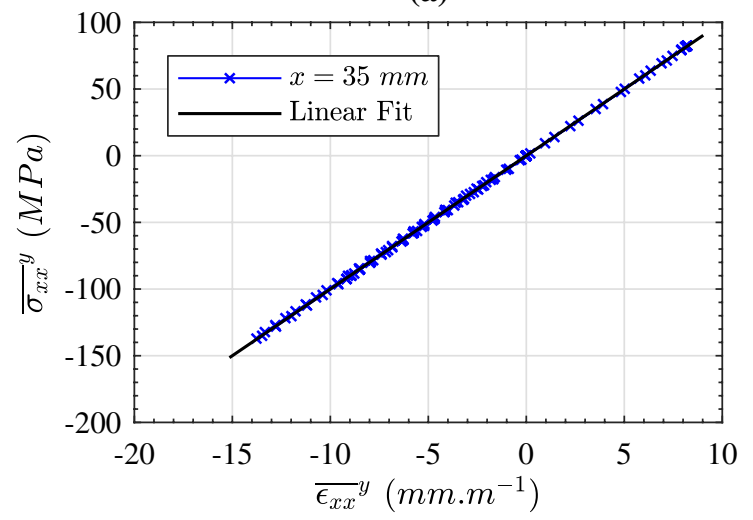

(b)

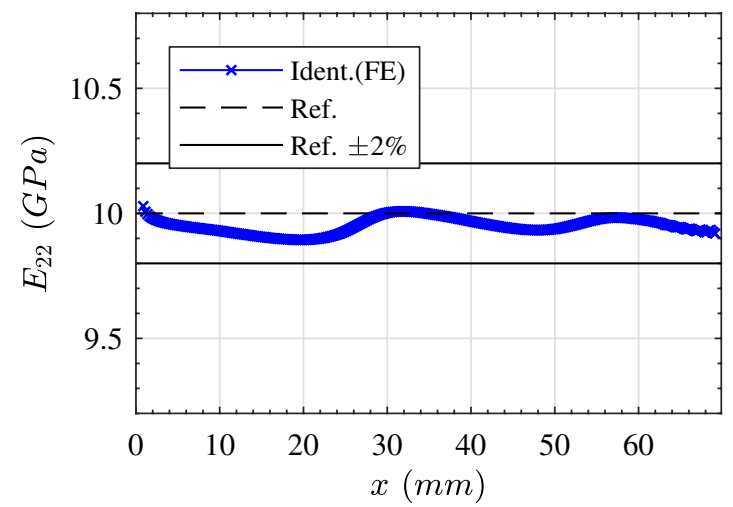

Fig. 9 a Stress-strain curve reconstructed from the 2D finite element model acceleration and strain fields near the specimen centre $x=35$ mm. $\mathbf{b}$ Identified elastic modulus from fitting stress-strain curves over specimen length for the finite model

(a)

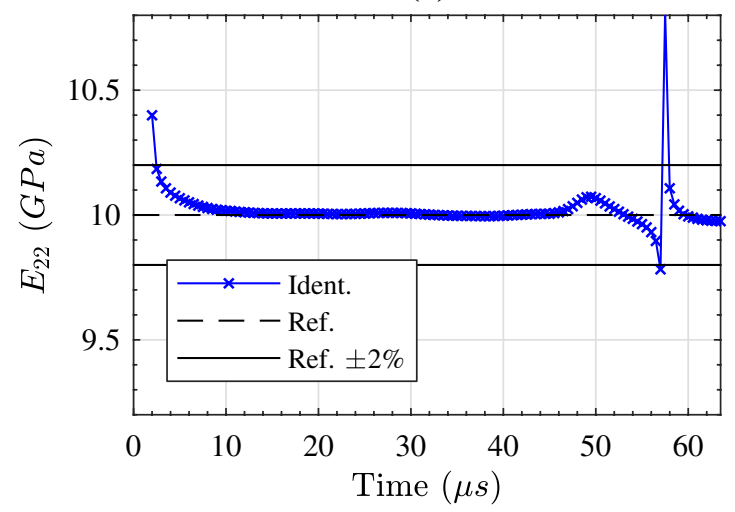

(b)

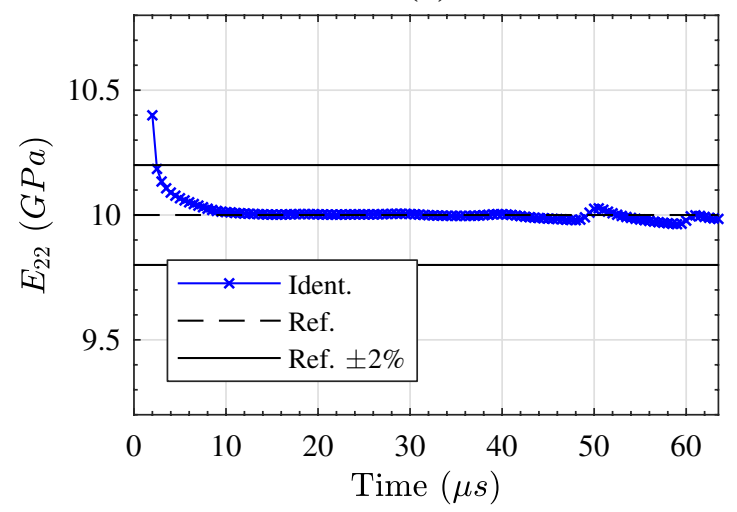

Fig. 10 a Identification over time for the manually defined virtual field applied to the $2 \mathrm{D}$ finite element model. $\mathbf{b}$ Identification over time for the special optimised virtual fields applied to the 2D finite element model 


\section{Experimental Methodology}

\section{Composite Material and Quasi-Static Tests}

The composite material used in this study was a unidirectional pre-preg tape with a low temperature cure epoxy matrix, Gurit SE70. The layup of the material was unidirectional with 15 layers $\left(\left[90^{\circ}\right]_{15}\right)$. The layers of composite pre-preg tape were mounted on a ground aluminium plate that acted as a mould. The composite was then cured in a vacuum bag under 1 bar pressure in an autoclave oven at $110^{\circ} \mathrm{C}$ for 50 minutes. The final cured plate had a thickness of $4.75 \mathrm{~mm}$ with a nominal fibre volume fraction of $55 \%$. Six specimens were machined from the plate using an irrigated diamond saw to the nominal in-plane dimensions of $70 \times 45$ $\mathrm{mm}$. The density of the material was measured using a micro balance and water immersion to be $1530 \pm 25 \mathrm{~kg} / \mathrm{m}^{3}$.

Quasi-static testing of the material was also undertaken using the same pre-preg material. For the quasi-static testing, six specimens were cut from a unidirectional plate $\left(\left[90^{\circ}\right]_{8}\right)$ cured using the same method described above. General testing conditions and specimen configuration conformed to the standard ISO 527-5:2009 [24]. All quasi-static testing was conducted on an Instron electromechanical load frame with a $50 \mathrm{kN}$ load cell. Strain measurements were performed using back-to-back unidirectional strain gauges mounted in the centre of the specimen and aligned with the long axis of the specimen. The strain rate for the quasi-static tests was $1 \times 10^{-4} \mathrm{~s}^{-1}$. It was not attempted to measure the minor Poisson's ratio $v_{21}$ here as it is very small and hard to measure accurately with this configuration. The results of the quasi-static testing are summarised in Table 3 and are provided as a comparison with the high strain rate data

presented later in the "Experimental Results and Discussion" section. The average quasi-static strength is low compared to manufacturer data for this pre-preg (nominally $\sigma_{F}=42$ $\mathrm{MPa}$ ). Subsequent microscopic observations showed that the cured pre-preg plate contained porosities, explaining the low quasi-static strength. The scope of this study is to develop the framework for the IBII test, not to fully characterise the properties of a particular composite system. Following this first validation of the IBII test method subsequent studies will need to be performed on well characterised composite systems.

\section{Impact Apparatus}

The IBII tests were carried out using a custom built gas gun facility at the University of Southampton. The waveguide and projectile were cylinders machined from an Aluminium

Table 3 Summary of experimental quasi-static testing results for transverse properties of the Gurit SE70 pre-preg conducted at a strain rate of $1 \times 10^{-4} \mathrm{~s}^{-1}$

\begin{tabular}{lll}
\hline Specimen & $\begin{array}{l}E_{22} \\
(\mathrm{GPa})\end{array}$ & $\begin{array}{l}\sigma_{22}^{F} \\
(\mathrm{MPa})\end{array}$ \\
\hline 1 & 7.43 & 34.3 \\
2 & 7.27 & 39.2 \\
3 & 7.31 & 39.6 \\
4 & 7.32 & 31.3 \\
5 & 7.20 & 37.9 \\
6 & 7.21 & 38.5 \\
Mean & 7.29 & 36.8 \\
SD & 0.085 & 3.32 \\
COV $(\%)$ & 1.17 & 9.01 \\
\hline
\end{tabular}

Note that SD represents the standard deviation and $\mathrm{COV}$ represents the coefficient of variation

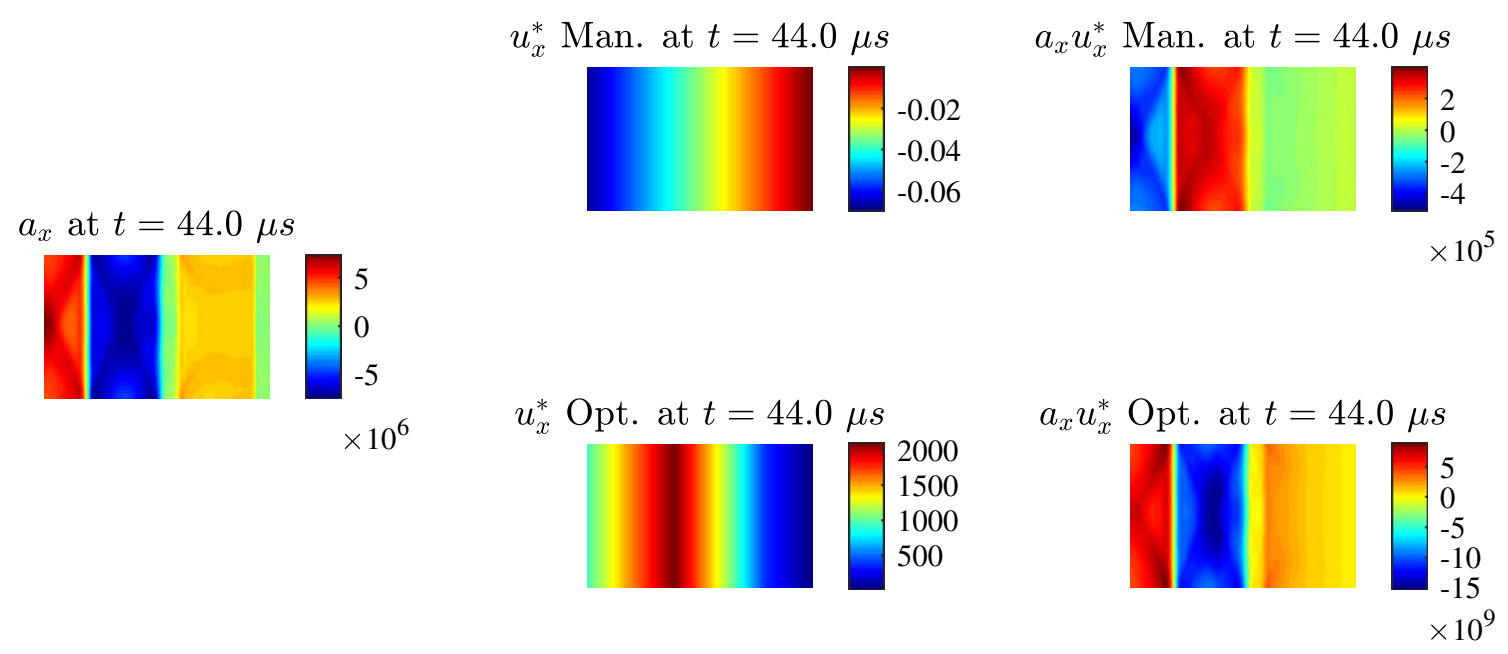

Fig. 11 Comparison of manual (Man.) and special optimised (Opt.) virtual fields at $t=44 \mu$ s applied to the 2D finite element simulation . The product of the virtual and kinematic fields shows that the optimised virtual fields follow the areas of high input signal. (Color figure online) 
6061-T6 rod with dimensions conforming to those selected in the parametric design sweep. The specimen was bonded to the cylindrical wave guide using cyanoacrylate glue. The waveguide was placed on a wedge-shaped foam stand $200 \mathrm{~mm}$ in front of the barrel exit. The foam stand does not directly attach to the waveguide, the waveguide is held in the centre of ' $v$ ' shaped stand by gravity. A custom mounting block was used to align the foam stand and wave guide with the gas gun barrel. A photograph of the impact chamber and a mounted specimen is shown in Fig. 12.

Two infra-red light gates were mounted in front of the exit of the barrel, these were connected to an Arduino Uno micro controller. The light gates were used to calculate the projectiles speed and to automatically trigger the photographic flash accounting for the flash rise time, $100 \mu$ s (Bowens Gemini Pro 1000). A copper contact trigger was used on the impacted face of the waveguide to trigger the camera. A delay of $11 \mu \mathrm{s}$ was used between the trigger event and image recording to account for the time taken for the input pulse to traverse the length of the waveguide before entering the sample. The gas gun reservoir pressure was set for a nominal impact speed of $35 \mathrm{~m} / \mathrm{s}$. The actual impact speed for all samples was $35.3 \pm 5.9 \mathrm{~m} / \mathrm{s}$ (mean \pm standard deviation). It should be noted that accurate control of the projectile speed is not required here as the response of the specimen is fully characterised by the measured full-field kinematics.

\section{Imaging and Full-Field Measurement Setup}

All experimental imaging was performed using a Shimadzu HPV-X ultra-high speed camera and a Sigma $105 \mathrm{~mm}$ macro lens. Additional imaging parameters used in this study are summarised in Table 4. The grid method was used for all

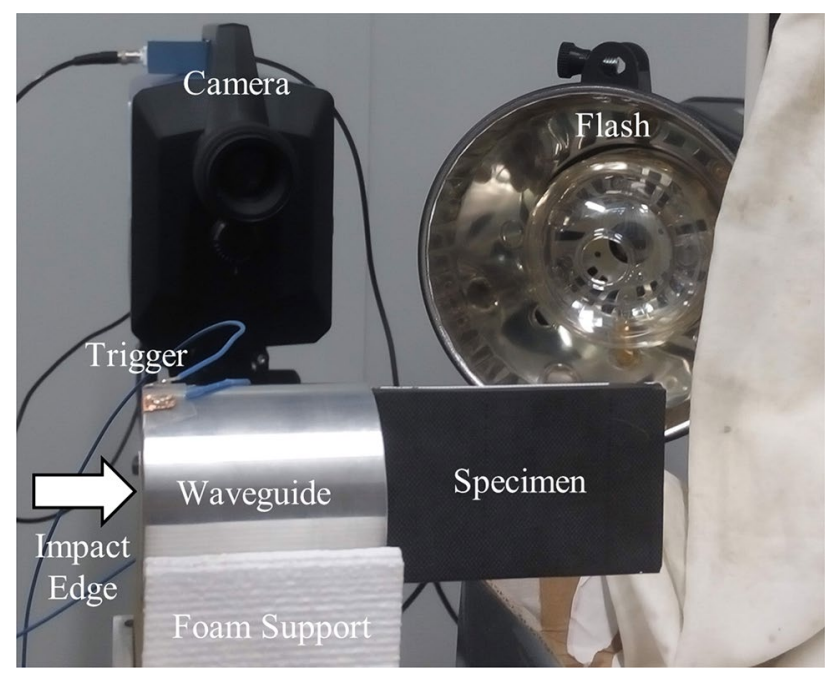

Fig. 12 Photograph of a transverse carbon fibre test specimen in-situ prior to testing full-field displacement measurements as it offers improved compromise between spatial and measurand resolution compared to DIC [25]. This is especially important here given the low number of pixels on the Shimadzu HPV-X $(400 \times 250$ pixels $)$. The main drawback of the grid method is that a regular grid needs to be deposited on the surface of the specimen. However, the regularity of the grid can be relaxed if an iterative displacement calculation procedure is used as described in ref. [26]. We note here that there is a similar requirement for optimal use of DIC as extremely large speckles require the use of large subsets, compromising spatial resolution and extremely small speckles cause aliasing. Thus, for optimal use of DIC strict control of speckle size is required and producing a high quality speckle pattern presents similar challenges to producing a regular grid. For this study, the grid method was used along with the iterative displacement calculation described in ref. [26]. Grids with a pitch of $0.9 \mathrm{~mm}$ were bonded to the specimens using the process outlined in ref. [27]. The grid pitch and sampling were selected such that the sample covered most of the camera field of view, maximising the use of available camera pixels.

There are practical issues associated with the technology used in the Shimadzu HPV-X camera, most notably, the low pixel fill factor. If not corrected for by slightly defocusing (i.e. blurring) the grid image, this can lead to a systematic error in the strain which manifests as an artificial fringe pattern in the strain fields $[28,29]$. This raises the question of 'how much should the image be blurred to remove the fill factor problem?'. Therefore, prior to each impact experiment, the image was blurred and an out-of-plane movement test was conducted. The out-of-plane movement test was performed by taking a series of static images, then moving the camera back $5 \mathrm{~mm}$ using a translation stage attached to the tripod. A second series of static images were then taken

Table 4 Summary of imaging, grid and smoothing parameters used for the experiments

\begin{tabular}{ll}
\hline Camera & Shimadzu HPV-X \\
Pixel array size & $400 \times 250$ \\
Total frames & 128 \\
Inter frame time & $0.5 \mu \mathrm{s}$ \\
Integration (shutter) time & $0.2 \mu \mathrm{s}$ \\
Grid pitch & $0.9 \mathrm{~mm}$ \\
Grid sampling & 5 pixels/period \\
Field of view & $72 \times 45 \mathrm{~mm}$ \\
Raw displacement resolution & $0.3 \mu \mathrm{m}$ \\
Spatial smoothing & Gaussian $25 \times 25$ pixels \\
Temporal smoothing & Savitsky-Golay, 3rd \\
& order over 17 frames \\
Strain resolution & $80 \mu \mathrm{m} / \mathrm{m}$ \\
Acceleration resolution & $6 \times 10^{5} \mathrm{~m} / \mathrm{s}^{2}$ \\
\hline
\end{tabular}


and, correlated to the first using the grid method. The outof-plane movement leads to an artificial in-plane hydrostatic strain because of the change in magnification. If the blurring is not sufficient to mitigate the fill factor problem, then fringes appear in the strain fields which should nominally be uniform spatially. This process is repeated until the fringes almost completely disappear from the strain fields. The camera is then moved back to its original position and another series of static images are taken prior to testing. For further information on the full-field measurement performance of ultra-high speed cameras with specific detail on the associated problems with the fill factor, the reader is referred to $[28,29]$.

\section{Experimental Data Processing}

The raw grey-level images were processed using an open source grid method processing code [26]. More detailed information on the grid method theory, practical implementation of the method and data processing can be found in a detailed review by Grédiac et al. [26]. In brief, the grid method uses a priori knowledge that the pattern on the specimen is regular and nominally sinusoidal. Signal processing algorithms can then be used to determine the phase at each camera pixel sampling of the grid. The phase is related directly to the displacement field of the specimen. Phase maps taken from the specimen surface can exhibit sharp discontinuities when the in-plane deformation is large. To account for this, spatial unwrapping is required. The algorithm used here was developed in ref. [30] and comes prepackaged with the grid processing code of Grédiac et al. [26]. Discontinuous jumps in the phase can also occur between frames (i.e. in time) if the rigid body motion of the test sample is larger than a grid pitch. For the impact test considered here, the rigid body motion is small between frames (much less than the grid pitch) and it increases monotonically. Therefore, the temporal discontinuities can be accounted for by adding integer multiples of the $2 \pi$ to the phase maps to ensure that the average displacement increases monotonically over the test duration. This is referred to as temporal unwrapping. The temporal unwrapping function used here was developed in-house. Subsequent processing of the full-field displacement maps was performed using custom-built Matlab programs (v R2015a).

Spatial and temporal smoothing were used to reduce the effects of camera noise and reach the required strain and acceleration resolutions. Smoothing filters were applied directly to the displacement fields before numerically differentiating in space (for strain) or in time (for acceleration). The numerical differentiation method was a centred finite difference with a forward or backwards difference at start or end of the data series (as implemented by the Matlab function gradient). For the strain calculation, only a spatial filter was used (i.e. no temporal smoothing) on the displacement field before numerical differentiation to obtain the strains. To obtain the acceleration fields only a temporal filter was used on the displacement fields (i.e. no spatial smoothing). The smoothed displacement fields were then numerically differentiated in time to obtain the velocity fields and then differentiated again with the same technique to obtain the acceleration. Relevant smoothing parameters are summarised in Table 4. For the Gaussian smoothing filter, problems arise on the borders of the data. These were dealt with by using the in-built symmetric padding option in Matlab.

While it is beyond the scope of the present work, it should be noted that the optimal method for determining filter parameters (and the resulting random and systematic errors) is to use image deformation simulations $[31,32]$. The underlying principle of this technique is described in detail in refs. [31-34]. Briefly, the idea behind this technique is to use displacement fields taken from a known solution to the underlying kinematics that encodes the desired constitutive parameters. If an analytical solution is available this can be used but for the dynamic case considered here the displacement fields can be taken from a finite element model. These displacement fields are then used to synthetically deform a set of images to replicate the spatial/temporal resolution and noise of the camera. These images can then be processed using exactly the same procedure as the experimental data (i.e. same number of pixels, frame rate, grid pitch etc.). The identified parameters can then be compared to the known constitutive parameters of the finite element model to determine the error. By sweeping a number of different processing parameters (i.e. different smoothing kernel sizes) with different copies of noise applied to the images the random and systematic error for each filter combination can be determined. Obviously, the largest smoothing kernels will result in the smallest random error but heavy smoothing will truncate the peaks in the signal leading to a large systematic error. The opposite is true for no smoothing. However, there is an optimum compromise between systematic and random error that can be determined using the image deformation procedure. Work is currently under way by the authors to develop an image deformation software pipeline to allow for the assessment of measurement error in identifying the stiffness and strength using the IBII technique described in this paper, but this is beyond the scope of this first paper.

Apart from smoothing to reduce random noise effects, there are further practical considerations associated with all full-field techniques, specifically the handling of missing data at the specimen border. For the grid method, one grid pitch worth of data at the specimen border is lost. The missing data on the border has been shown to have an effect on material models identified using the VFM combined with DIC [35]. However, in the study by Rossi et al. [35], it was shown that padding or extrapolating the data can lead to a 
significant improvement in the identified parameters. For the impact test considered here, the missing data on the specimen border will affect the identification procedure. In order to address this problem, the strain and acceleration data were linearly extrapolated over the missing data region using the extrapolation option of the Matlab function interp. The extrapolation was performed after all smoothing and numerical differentiation procedures.

The raw images, data processing program and associated output are provided for all tested specimens in the data repository detailed at the end of the manuscript.

\section{Experimental Results and Discussion}

\section{Full-Field Measurement Results}

For all subsequent images and analysis presented, specimen 4 was selected as a typical representation of all specimens tested. Note that additional images and videos are provided for all tested specimens in the data repository mentioned at the end of the manuscript. The full-field displacement maps for specimen 4 are shown in Fig. 13 for the approximate time that the compressive wave is halfway through the sample $(t=24.5 \mu \mathrm{s})$, and at a time after the wave has reflected becoming tensile ( $t=52 \mu \mathrm{s})$. The corresponding acceleration and strain maps are shown in Figs. 14 and 15 respectively.

The acceleration map shown in Fig. 14a clearly shows the front and back edge of the input stress pulse. The acceleration maps for $a_{x}$ show peaks on the order of $10^{6} \mathrm{~g}$. The experimental strain maps also confirm that the magnitude of the $\varepsilon_{y y}$ strains are significantly lower (approximately one order of magnitude) than the $\varepsilon_{x x}$ strains. For the case of the $\varepsilon_{y y}$ strains shown in Fig. 15c, d, it is clear that the signal is much noisier than for the $\varepsilon_{x x}$ strains. This provides experimental evidence to validate the assumption that the contribution of the $\varepsilon_{y y}$ strain is small when formulating the constitutive law in the "Constitutive Model and Assumptions" section. The maps of the $\varepsilon_{x x}$ strain rate are shown in Fig. 16. These strain rate maps are heterogeneous and show that different regions of the material experience different strain rate amplitudes. It is difficult to gain a thorough understanding of a dynamic experiment from static images alone therefore, videos of the kinematic fields are provided for each specimen in the data repository associated with this paper.

From the surface average of the acceleration field the average loading force on the impact edge can be reconstructed: $\bar{F}_{x}^{y}=m_{s} \bar{a}_{x}^{S}$ where $m_{s}$ if the mass of the sample and $\bar{F}_{x}^{y}$ is the average force applied to the sample at the impact edge. The loading pulse for specimen 4 is shown in Fig. 17 showing peak loads on the order of $15 \mathrm{kN}$ in compression. The processed full-field acceleration and strain data can now be used to identify the stiffness and strength using the methods described in the "Concept Design and Theory" section. The stiffness identification is presented in the following section "Stiffness Identification" while the strength identification is presented in the "Strength Identification" section.
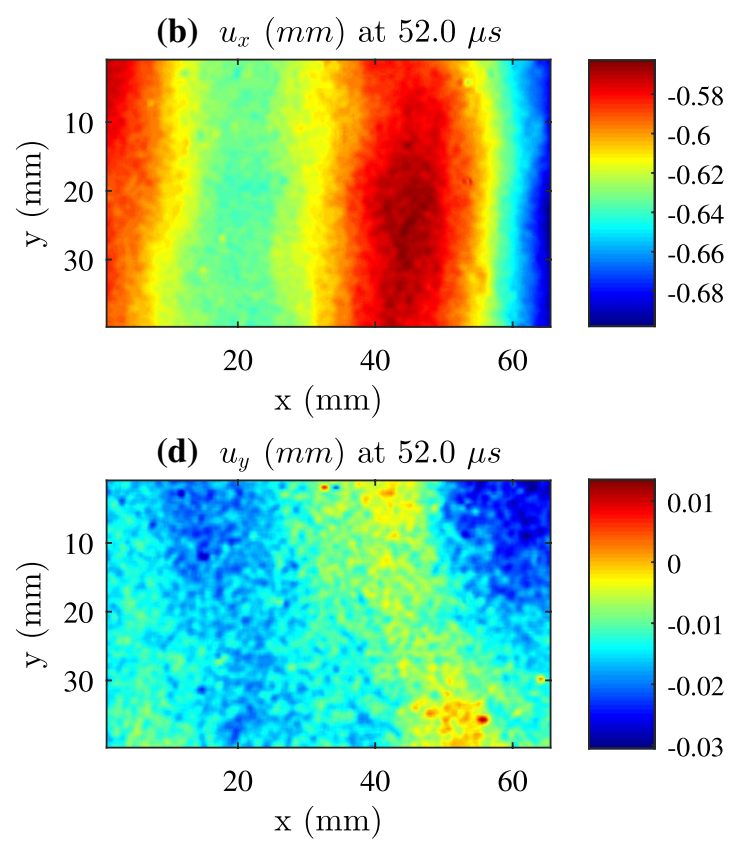

Fig. 13 Experimental displacement fields for specimen 4. a, $\mathbf{b} u_{x}$ and $\mathbf{c}, \mathbf{d} u_{y}$ at times $24.5 \mu \mathrm{s}$ and $52 \mu$ s. (Color figure online) 

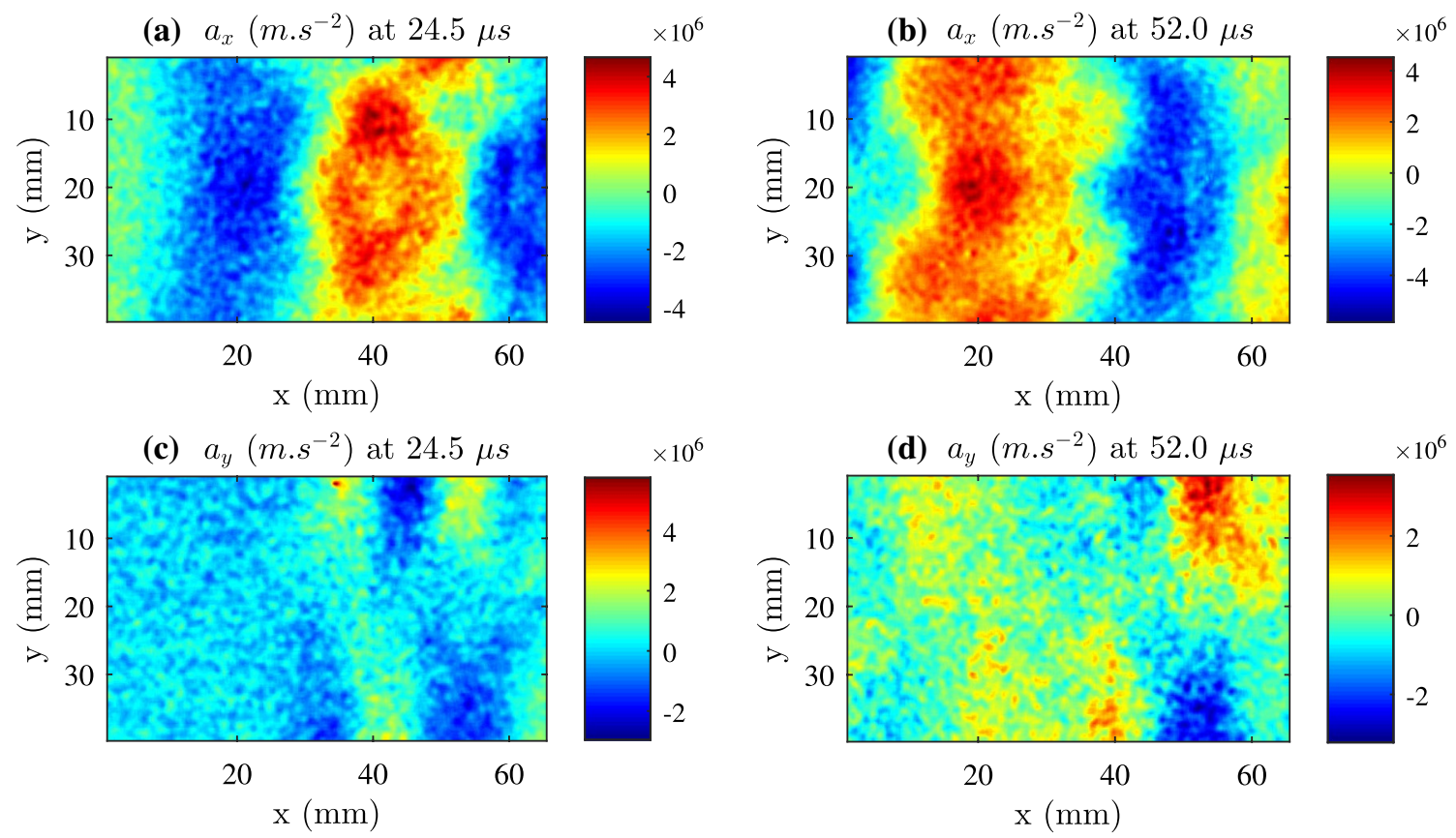

Fig. 14 Experimental acceleration fields for specimen 4. a, $\mathbf{b} a_{x}$ and $\mathbf{c}, \mathbf{d} a_{y}$ at times $24.5 \mu$ s and $52 \mu$ s. (Color figure online)

\section{Stiffness Identification}

The stress-gauge equation was used to calculate the average stress ${\overline{\sigma_{x x}}}^{y}$ from the acceleration fields for all six specimens (eq. 16). The peak average compressive stress for specimen 4 was $76 \mathrm{MPa}$. The experimental compressive stress is well below the given design criterion of an input pulse below $200 \mathrm{MPa}$. Therefore, the compressive pulse is highly unlikely to cause any compressive damage and corrupt the stiffness identification.

The mean axial stress was used to plot stress-strain curves for different sections along the specimen length. The transverse stiffness $E_{22}$ was then identified by linearly fitting the stress-strain curve up to the point of maximum compressive stress in each section. This avoided using any tensile response as some micro-damage may appear early and compromise the measurement of the initial stiffness of the material. Stress-strain curves for several sections along the length of specimen 4 are shown in Fig. 18a. The identified elastic modulus as a function of position for specimen 4 is also shown in Fig. 18b. Similarly, the identified modulus as a function of length is shown for all six specimens in Fig. 19. Analysis of Figs. 18 and 19 shows that the data near the specimen edges is of poor quality. This is due to several factors including: 1) the data extrapolation on the borders; 2) edge effects in the strain coming from spatial smoothing; and 3) physical defects in the grids at the edges leading to outliers in the kinematic data. If the modulus data at both specimen ends is ignored, a representative modulus can be obtained by taking the average over the middle $50 \%$ of the specimen. For specimen 4, shown in Fig. 18, this results in an identified transverse modulus of $7.9 \mathrm{GPa}$. The method described previously was used to identify the transverse modulus for all tested specimens. The results are summarised in Table 5. The average modulus identified over all specimens was $7.9 \mathrm{GPa}$ with a coefficient of variation (COV) of 3.2\%. The coefficient of variation for the stiffness measurement using this approach is low considering the low pixel count of the camera used. The COV also compares well with that obtained in the quasi-static testing campaign results in Table 3.

The transverse elastic modulus was also identified using the manual and special optimised virtual fields. The transverse stiffness is shown as a function of time for both the manual and optimised fields in Fig. 20 for specimen 4. A mesh convergence study was conducted for the special optimised virtual fields. The stiffness identification was found to stabilise with a virtual mesh of $5 \times 1$ elements. Therefore, a $5 \times 1$ virtual mesh was used was used for all specimens. The identification over time for all specimens is shown in Fig. 21. Note that in Fig. 21, the identification has been stopped after the appearance of a macro crack on the strain maps. The identification for all specimens is stable over a relatively large portion of the test, apart from several time frames at which the acceleration signal is low. When the wave first enters the specimen, there is minimal acceleration signal leading to an unstable identification. Once the wave has fully entered the specimen, the identification becomes stable. A 

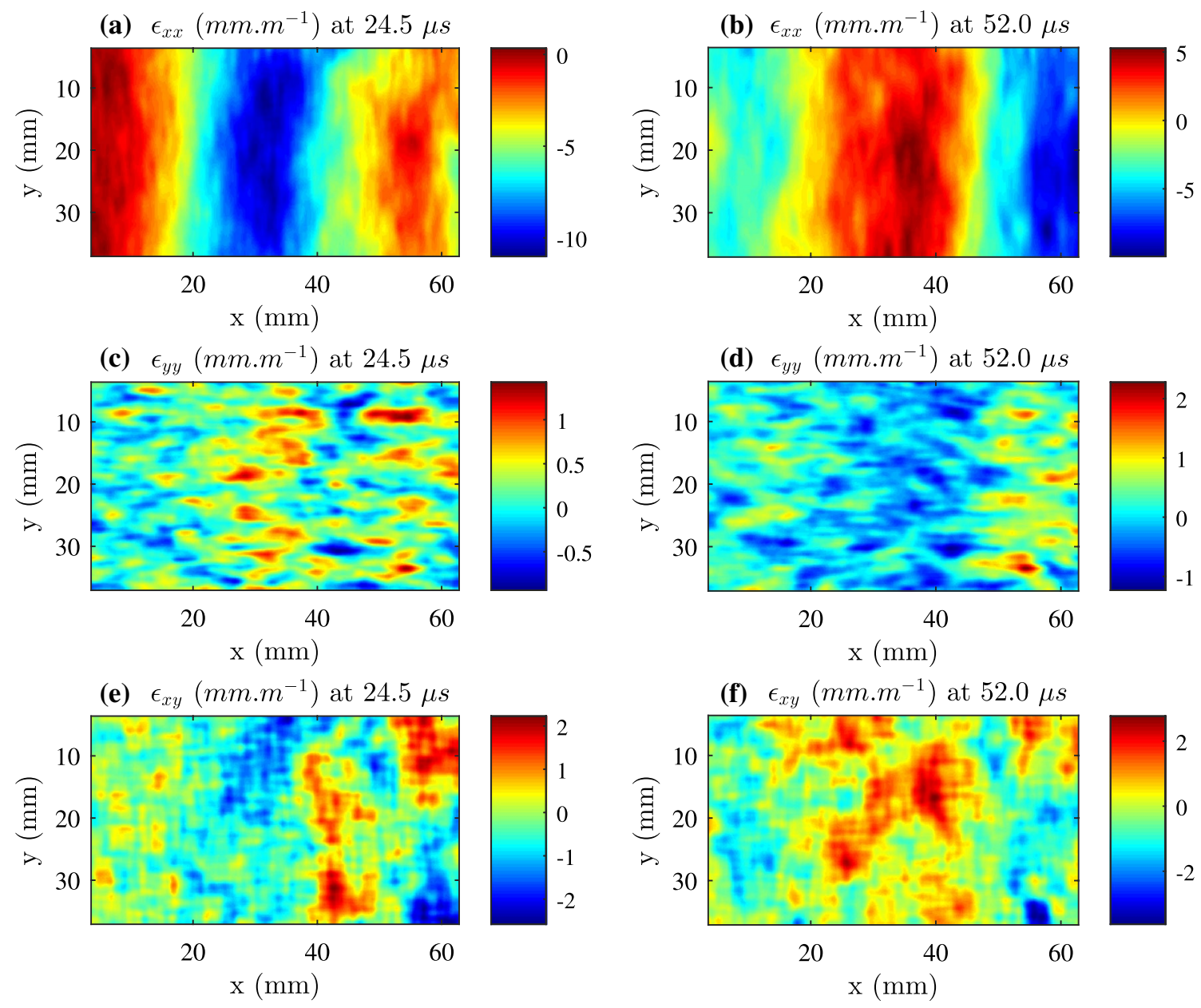

Fig. 15 Experimental strain fields for specimen 4. a, b $\varepsilon_{x x} ; \mathbf{c}, \mathbf{d} \varepsilon_{y y}$; and $\mathbf{e}, \mathbf{f} \varepsilon_{x y}$ at times $24.5 \mu$ s and $52 \mu$ s. (Color figure online)
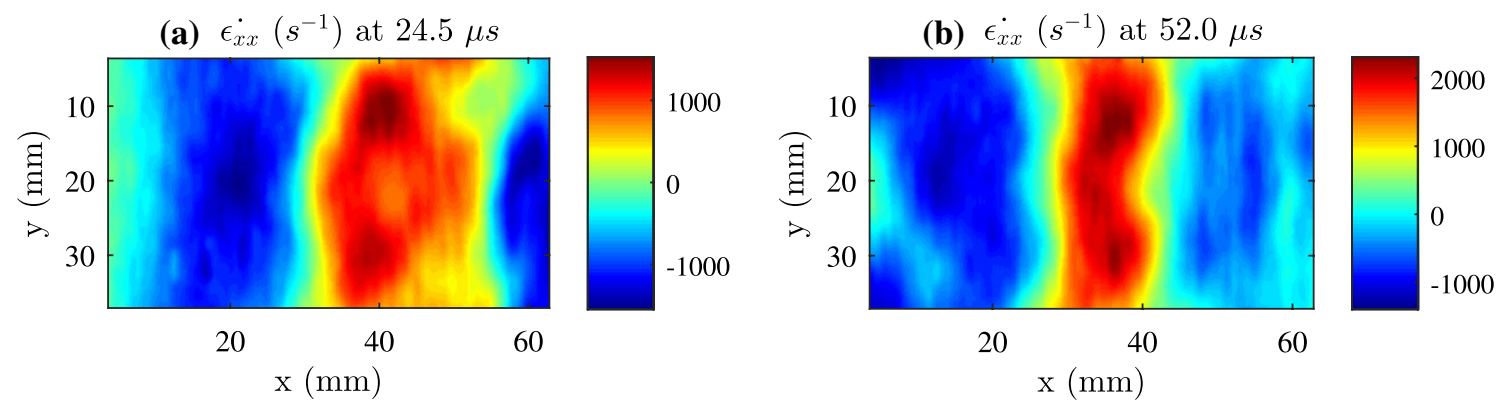

Fig. 16 Experimental strain rate fields for specimen 4. $\varepsilon_{x x}^{*}$ at times $\mathbf{a} 24.5 \mu \mathrm{s}$ and $\mathbf{b} 52 \mu \mathrm{s}$. (Color figure online)

similar instability occurs again once the wave reflects off the free edge leading to low acceleration signal. It is also shown in Figs. 20, 21 that the identified stiffness drops at the end of the test. This is due to the specimen cracking and failing, rendering the virtual fields analysis non-physical (i.e. nonphysical strain information due to cracks). In order to make the modulus identified with the the VFM comparable to the stress-gauge equation, the time frames for the initial compressive loading is used. For this purpose, a representative modulus was identified by averaging between 15 and $30 \mu \mathrm{s}$. The average transverse modulus identified for specimen 4 is 7.8 GPa using both the manual and optimised virtual fields. The stiffness identified for each specimen using the VFM is summarised in Table 5. The manual and optimised VFM 


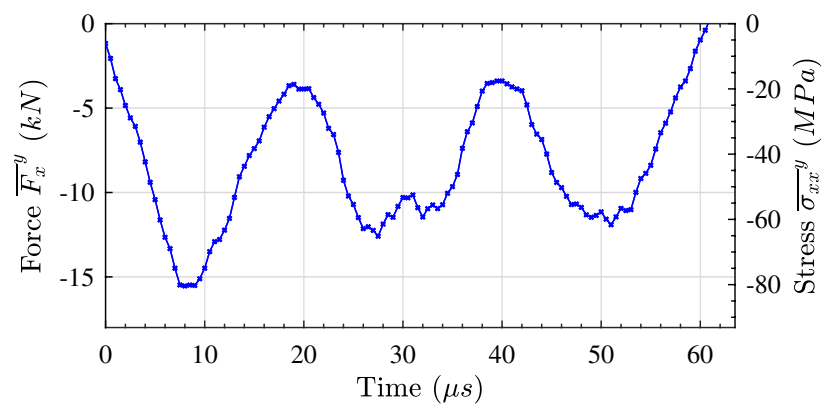

Fig. 17 Experimental loading pulse applied to specimen 4 reconstructed using the average acceleration over the field of view. Note the multiple loading pulses coming from the wave speed mismatch between the projectile and the specimen

Table 5 Identified transverse elastic modulus from experimental data using the stress gauge approach (SG), the manual (VF Man.) and optimised (VF Opt.) virtual fields method: including the mean, standard deviation (SD) and coefficient of variation (COV)

\begin{tabular}{|c|c|c|c|}
\hline Specimen & $\begin{array}{l}E_{22} \\
\mathrm{SG} \\
(\mathrm{GPa})\end{array}$ & $\begin{array}{l}E_{22} \\
\text { VF Man. } \\
(\mathrm{GPa})\end{array}$ & $\begin{array}{l}E_{22} \\
\text { VF Opt. } \\
(\mathrm{GPa})\end{array}$ \\
\hline 1 & 8.3 & 9.9 & 8.2 \\
\hline 2 & 7.8 & 8.3 & 7.9 \\
\hline 3 & 8.2 & 8.4 & 8.2 \\
\hline 4 & 7.8 & 7.8 & 7.8 \\
\hline 5 & 7.6 & 8.0 & 7.6 \\
\hline 6 & 7.9 & 9.0 & 7.1 \\
\hline Mean & 7.9 & 8.6 & 7.8 \\
\hline SD & 0.3 & 0.8 & 0.4 \\
\hline $\operatorname{COV}(\%)$ & 3.2 & 8.8 & 5.5 \\
\hline \% Diff. to QS & +8.8 & +18 & +7.0 \\
\hline
\end{tabular}

Percentage difference with respect to the quasi-static (QS) value is also provided

values show distinct differences when compared across all specimens (see Fig. 21a, b). The reason for this is that the optimised virtual fields method follows the areas of the kinematic fields that contain the largest signal whereas the manual fields act like a static spatial filter regardless of the input signal. Therefore, it is expected that the optimised virtual fields results will be more robust across all time frames, which is what is seen here.

The identification of the transverse elastic modulus is quite consistent between specimens and identification methods except for the manually defined virtual fields. Comparing the results to the quasi-static reference modulus (7.29 GPa) shows an increase of $8.8 \%$ when compared to the modulus obtained with the stress-gauge equation, and an increase of $7.0 \%$ when compared to the modulus obtained using the optimised virtual fields. This increase in elastic modulus is expected for the transverse properties of a unidirectional composite as the transverse properties are dominated by the matrix material. For the tested material, the matrix is epoxy. Polymers such as epoxy show some strain rate dependence and are generally thought to have increasing stiffness with increasing strain rate [6,7]. This increase in stiffness with increasing strain rate is due to a reduction in polymer chain mobility with increasing strain rate $[6,7]$.

It is difficult to compare the results of this study with previous studies using the SHPB technique at this strain rate. As previously mentioned, the influence of inertial effects only allow an apparent modulus to be obtained with the SHPB [8, 9]. Further complications arise in comparing the transverse properties of composites as they are highly dependent on the matrix material. Differences in the chemical composition of the epoxy resin will lead to differences in the strain rate dependence. For example, more or less cross-linking will change the polymer chain mobility and hence, the strain rate dependence of the material. Here, a tentative comparison can be made with the results of a study by Kwon et al. [36]. Kwon et al. used a high speed hydraulic test machine coupled with DIC to analyse the strain rate dependence of CFRP at strain rates between 0.001 and $100 \mathrm{~s}^{-1}$. The results of this study show an increase of $25 \%$ in the transverse modulus from the lowest to highest strain rate tested. This is significantly higher than the percentage increase found in this study. A possible reason for this could be the difference in the matrix material of the composites tested, or experimental difficulties in measuring the applied force at higher strain rates.

When identifying the high strain rate properties of a material, it is important to consider the strain rate at which the material is tested. While this task is trivial for monotonic quasi-static testing, it is more complicated for high rate dynamic testing. The strain rate for high strain rate dynamic tests will inevitably be heterogeneous and transient due to inertial effects (i.e. stress wave propagation). This is evident when considering the strain rate maps in Fig. 16. This raises the question: what strain rate corresponds to the identified modulus values? While there is no definitive answer to this question at this stage, it is possible to extract the peak width averaged strain rate for each specimen to give an indication of the strain rates achieved. A summary of the peak tensile and compressive strain rates for each specimen is given in Table 6. Note that these peak strain rate values are the maximum strain rates before the first macro crack appears on the raw strain maps. The width averaged strain and strain rate history for several axial sections of specimen 4 is also provided in Fig. 22. Note that these axial sections correspond to the stress-strain curves shown in Fig. 18a. Analysis of Fig. 22 shows that for most of the test, when the strain magnitude is moderate the strain rate tends to be high. Therefore it may be possible to define an "effective" strain rate using 
(a)

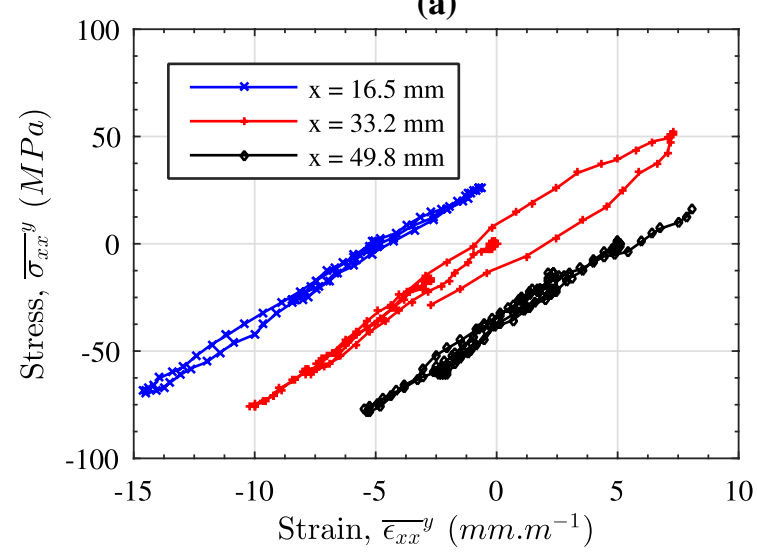

Fig. 18 a Stress-strain curves at $25 \%, 50 \%$ and $75 \%$ of the specimen length from the free edge. Note that all stress-strain curves start near the origin but have been offset by $5 \mathrm{~mm} / \mathrm{m}$ for clarity. b Transverse (b)

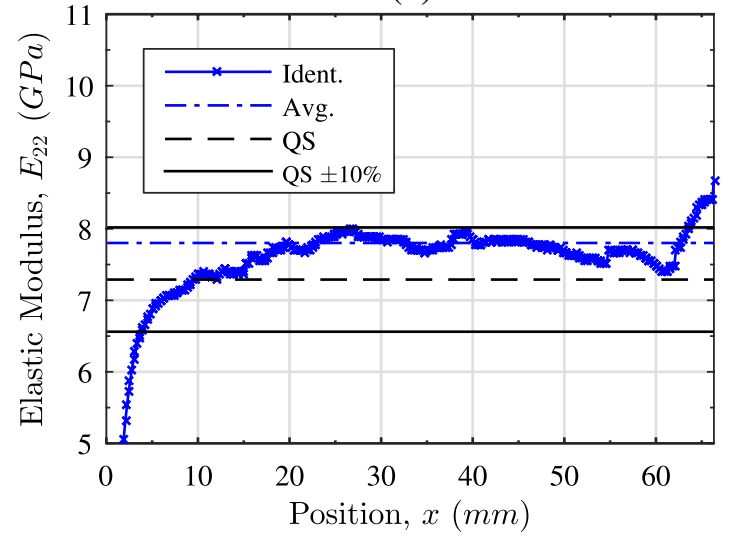

elastic modulus identified using the stress-gauge equation as a function of specimen length including comparison to the quasi-static value (QS). Note that this is experimental data taken from specimen 4

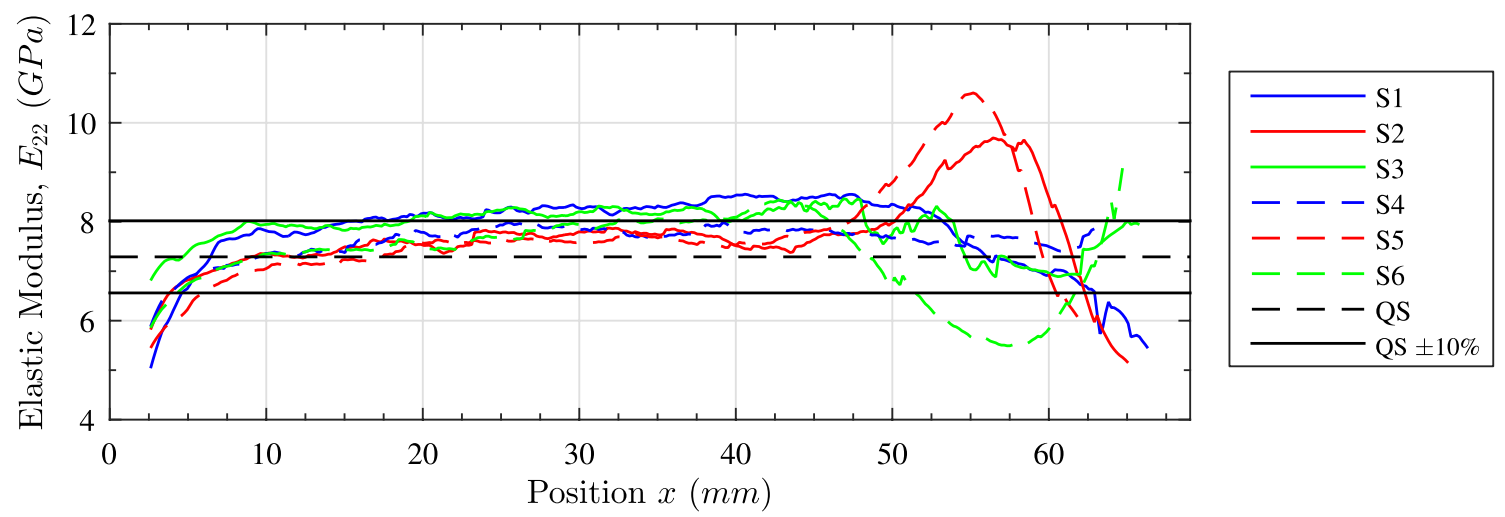

Fig. 19 Transverse modulus identified using the stress-gauge equathat the extrapolated data on the specimen ends has been removed. tion for all six specimens as a function of the specimen length. The Data markers have been removed for clarity. (Color figure online) quasi-static (QS) value is provided for comparison purposes. Note

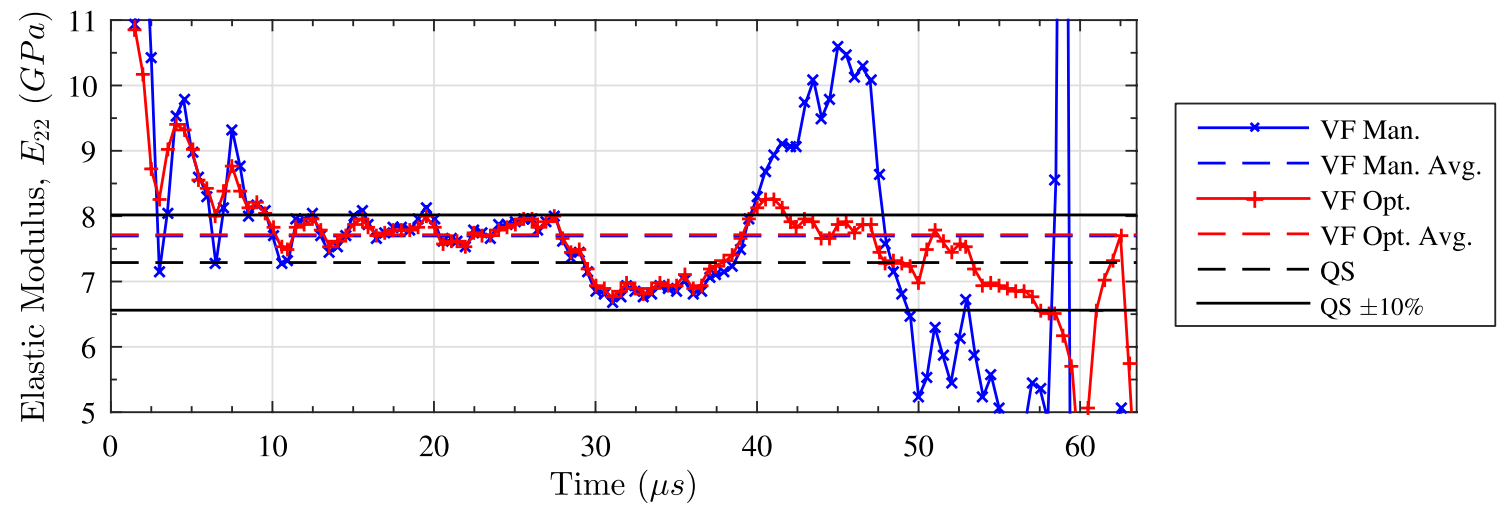

Fig. 20 Transverse elastic modulus identified from experimental data using the manual (VF Man.) and optimised virtual fields (VF Opt.) method as a function of time for specimen 4. The quasi-static (QS) value is provided for comparison purposes. (Color figure online) 

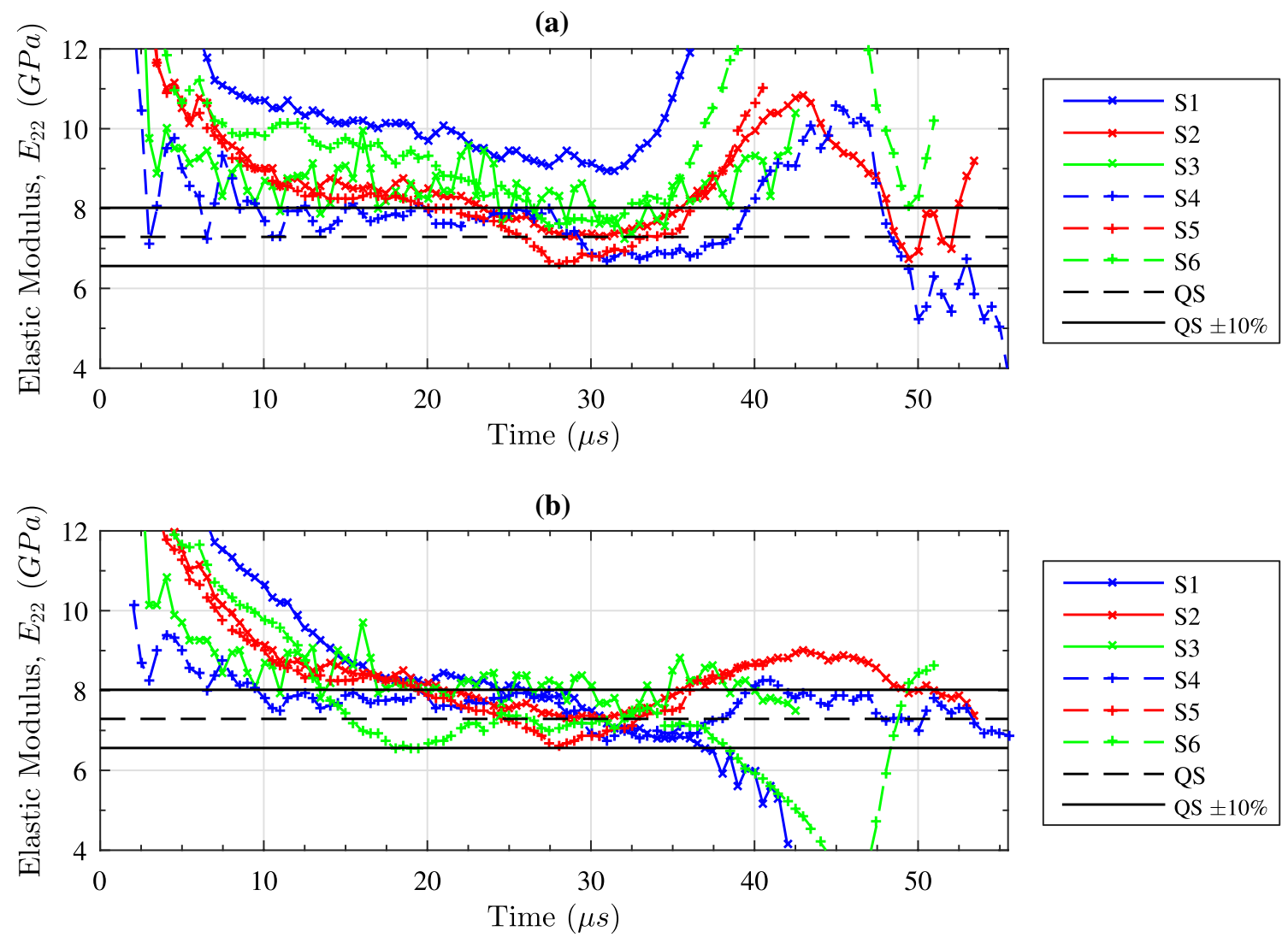

Fig. 21 Transverse elastics modulus as a function of time for all six experimental specimens using a the manual virtual field and $\mathbf{b}$ the optimised virtual fields method. Note that the first five time steps have been removed as there is inadequate spatial information for reli-

Table 6 Peak tensile (tens.) and compressive (comp.) width averaged strain rate ${\overline{\varepsilon_{x x}}}^{y}$ from the experimental data

\begin{tabular}{lll}
\hline Specimen & $\begin{array}{l}\text { Peak tens. } \\
{\overline{\varepsilon_{x x}^{-}}}^{-y}\left(\mathrm{~s}^{-1}\right)\end{array}$ & \begin{tabular}{l}
${\overline{\varepsilon_{x x}^{-}}}^{-y}\left(\mathrm{~s}^{-1}\right)$ \\
\hline 1
\end{tabular} \\
2200 & -1800 \\
2 & 2700 & -2200 \\
3 & 3100 & -2800 \\
4 & 2200 & -1800 \\
5 & 2900 & -2400 \\
6 & 3100 & -2500 \\
\hline
\end{tabular}

this data. However, this is beyond the scope of the present work. For all specimens, the peak width averaged strain rate is on the order of $2000 \mathrm{~s}^{-1}$. $E_{22}$ is not very rate sensitive here, with only an $8 \%$ increase between quasi-static and a few $1000 \mathrm{~s}^{-1}$, so this is not really critical here. If a material exhibited very strong strain rate sensitivity, then a strain rate sensitive material model could be included in the VFM able identification. Additionally, the identification has been stopped after the first macro crack appears on the strain maps. (Color figure online)

formulation to be identified. This may have to be implemented in the future for certain materials as required.

\section{Strength Identification}

In order to identify the strength, there are three separate issues to address: (1) determining the location of failure; (2) determining the time of failure; and (3) deriving the failure stress at the time of fracture. The first problem is addressed by analysis of the raw (i.e. unsmoothed) strain maps throughout the test. Fig. 23 shows the raw strain map for specimen 4 once a macro crack has formed. Here the crack is clearly shown as an area of (non-physical) strain concentration.

The second and third issues present some interesting challenges when using an ultra-high speed camera with a low pixel resolution. It is possible that the material fails and a crack starts to open before it is directly observable on the strain maps. Further, it is also possible that the material will damage or plastically deform before a macro crack forms. Given that plasticity in polymers tends to be suppressed with 
(a)

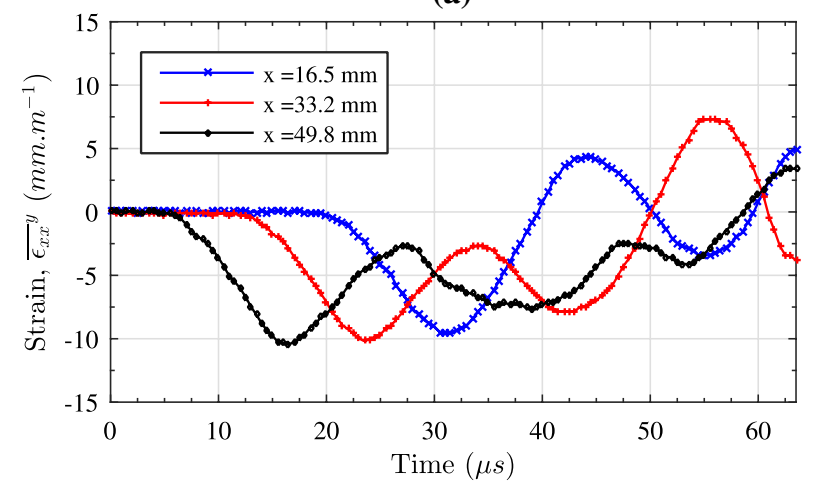

(b)

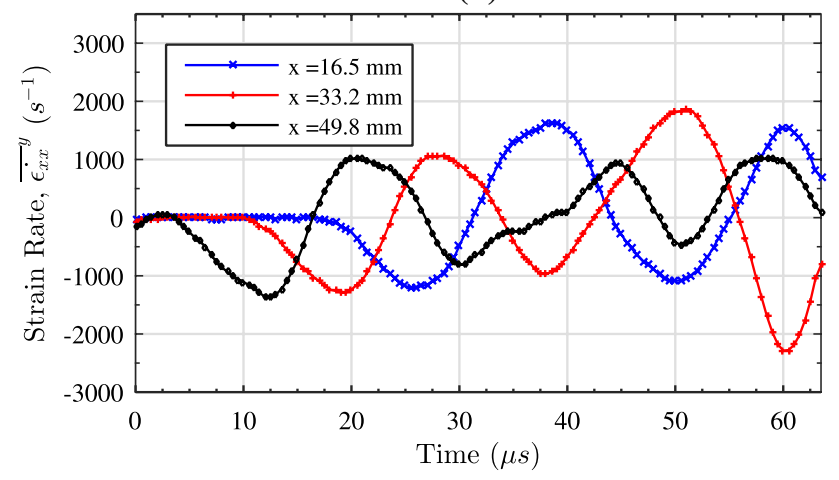

Fig. 22 Width averaged experimental data for specimen 4. a Strain $\bar{\varepsilon}_{x x}^{y}$ and $\mathbf{b}$ strain rate $\bar{\varepsilon}_{x x}^{-y}$ history corresponding to the stress-strain curves in Fig. 18a

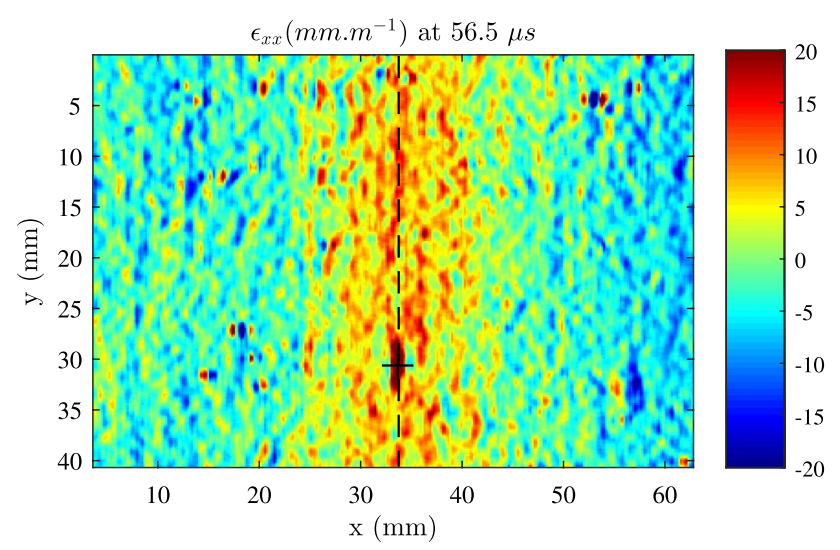

Fig. 23 Raw unsmoothed strain map $\left(\varepsilon_{x x}\right)$ from the experimental data of specimen 4 showing the fracture location. (Color figure online)

increasing strain rate it is more likely that a damage process is occurring [6]. Therefore, accurately determining the failure time becomes challenging. It is possible to partially resolve the problem of identifying the time of failure by using two different types of stress information. Specifically, the mean stress calculated from the acceleration and the stress calculated from the identified material model can be compared at the fracture location. When the material starts to form damage, the stress calculated using the strains will diverge from the stress calculated using the accelerations. The point at which these two measures diverge can be used to identify the the point of damage onset (or plasticity) in the material. After a critical amount of damage is formed, a macrocrack will nucleate and the material will fracture. Here, the fracture was assumed to occur at the maximum tensile stress measured using the acceleration after the two different methods of estimating the stress have diverged. The fracture time is then the frame at which the maximum stress occurs.

In order to compare both stress measures in the experimental data, the mean stress over a virtual gauge area of 11 (x) by 21 (y) pixels was used. The spatial average over the virtual gauge area will be denoted using a superscript $A$ following the overline notation, e.g. ${\overline{\sigma_{x x}}}^{A}(L S G)$ denotes the average stress in the virtual gauge area using the linear stress-gauge equation (eq. 38). This gauge area is shown on the stress maps in Fig. 24a, b. For the purpose of calculating the stress from the strains, the average elastic modulus from the stress-gauge and special optimised virtual field was used. Note that the individually identified elastic modulus was used for each specimen. The divergence of the two stress measures is shown clearly in Fig. 24c, d. From Fig. 24c, the two stress measures begin to diverge just after $53.0 \mu \mathrm{s}$ and the maximum stress calculated from the acceleration occurs at $55.5 \mu \mathrm{s}$. The divergence of these measures is shown as non-linear behaviour in the stress-strain curve over the virtual gauge region in Fig. 24d. This non-linear behaviour prior to failure suggests that there may be plastic deformation or a damage process in the composite prior to the formation of a macro crack. However, the stress being derived from acceleration, this apparent softening could also come from temporal 'leakage' from the crack as the acceleration is temporally smoothed. This will need to be investigated further in the future using image deformation simulations. Using the average stress in the virtual gauge area from the linear stress gauge equation, the failure strength was identified to be $52 \mathrm{MPa}$ (this point is highlighted using a red cross in Fig. 24d).

It was possible to identify the transverse tensile strength for specimens 1-5. The identified strength for each of these specimens is summarised in Table 7. For comparison purposes, the strength derived using the standard stress-gauge equation is also provided in Table 7. It can be seen that both values are very consistent for specimens $3-5$, but differ significantly for specimens 1 and 2 . This shows that the LSG approach provides a more flexible stress measure to accommodate for some stress non-uniformity in the lateral direction. The strength values obtained through the LSG approach are very consistent and present a comparable $\mathrm{COV}$ to the quasi-static results. This is certainly the result 


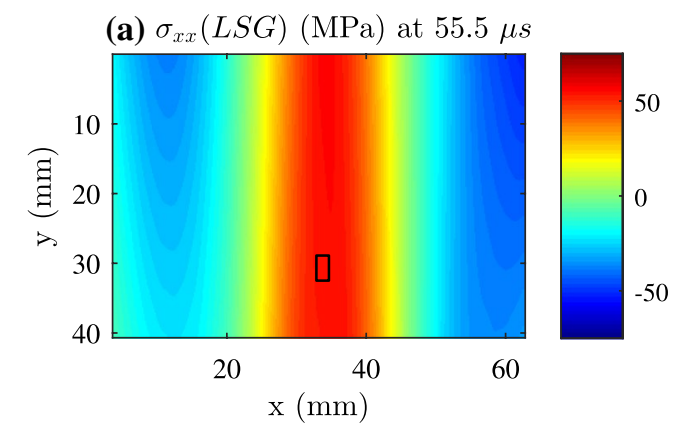

(c)

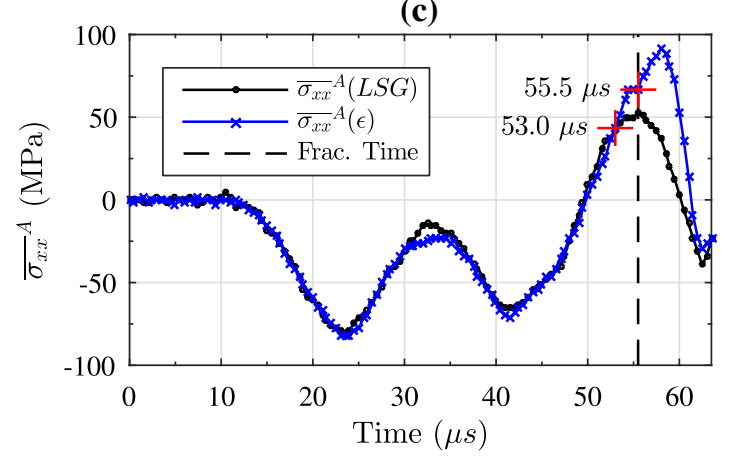

Fig. 24 Strength identification diagnostics for the experiment data from specimen 4. a Approximate stress field at the identified fracture time using the linear stress-gauge equation. b Approximate stress field at the identified fracture time using the strain and constitutive law. The virtual gauge area is shown as a black rectangle in a, b. c

of the very neat and consistent way of loading the material in tension through the waves while the quasi-static results will be more affected by possible specimen bending in the test machine. Additionally, the comparison of the average stress in the gauge area calculated using the acceleration ${\overline{\sigma_{x x}}}^{A}(L S G)$ and the strains ${\overline{\sigma_{x x}}}^{A}(\varepsilon)$ is shown in Fig. 25. For specimens $1-5$ both stress metrics are reasonably consistent up to the point of fracture.

Unfortunately, it was not possible to identify the transverse tensile strength for specimen 6 . The reason for this was due to a significant inconsistency between the stress distribution predicted using the stress-gauge equation and the stress distribution reconstructed using the identified constitutive law. This inconsistency was only evident after the wave had reflected. Note that this does not affect the identified elastic modulus for this specimen as the modulus identification procedure only uses the portion of the test prior to the wave reflecting and becoming tensile (i.e. the portion of the test where both measures of stress are consistent). It is unclear why this divergence occurred. However, it is suspected that through-thickness dispersion following the wave reflection may be responsible. This could be due to slight inconsistencies in machined specimen geometry (e.g. non-square edges through-thickness). This suggests that specimen machining procedures are critical for this type of test. To confirm that (b) $\sigma_{x x}(\epsilon)(\mathrm{MPa})$ at $55.5 \mu \mathrm{s}$

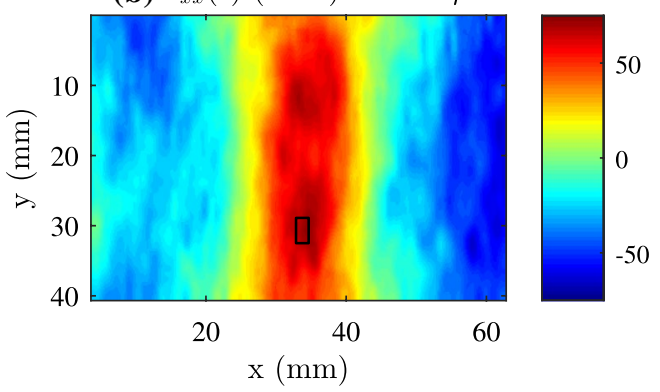

(d)

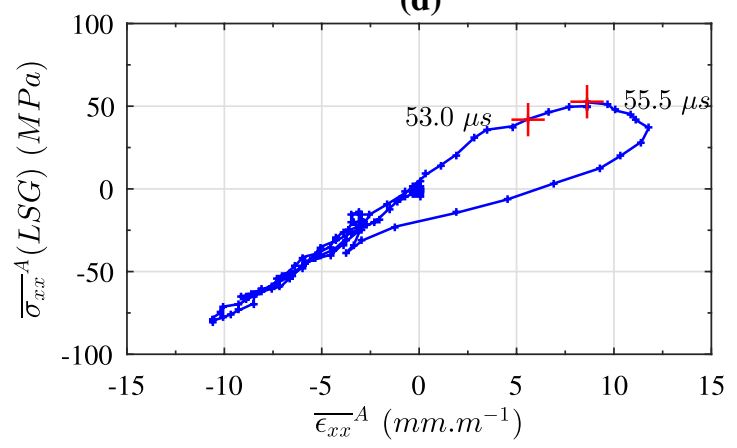

Average stress in the virtual gauge area using the linear stress-gauge equation ${\overline{\sigma_{x x}}}^{A}(L S G)$ and stress calculated from the strains $\bar{\sigma}_{x x} A(\varepsilon)$. d Stress-strain curve over the virtual gauge area at the fracture location. (Color figure online)

through-thickness dispersion is responsible for these inconsistencies, back-to-back imaging of the sample is required. Future experiments are planned to assess this phenomenon.

Table 7 Identified transverse tensile strength for all experimental specimens

\begin{tabular}{lll}
\hline Specimen & $\begin{array}{l}\text { Strength } \\
\bar{\sigma}_{x x}^{F}(S G)\end{array}$ & $\begin{array}{l}\text { Strength } \\
(\mathrm{MPa})\end{array}$ \\
& $\bar{\sigma}_{x x}{ }^{F}(L S G)$ \\
& 27 & $(\mathrm{MPa})$ \\
\hline 1 & 46 & 55 \\
2 & 58 & 69 \\
3 & 53 & 58 \\
4 & 51 & 52 \\
5 & - & 54 \\
6 & 47 & - \\
Mean & 12 & 58 \\
SD & 25 & 6 \\
COV (\%) & +28 & 11 \\
$\%$ Diff. to QS & & +57 \\
\hline
\end{tabular}

The strength has been calculated using the stress-gauge equation $\left({\overline{\sigma_{x x}}}^{F}(S G)\right)$ and the linear stress-gauge equation $\left({\overline{\sigma_{x x}}}^{F}(L S G)\right)$. The percentage difference with respect to the quasi-static (QS) value is also provided for comparison 

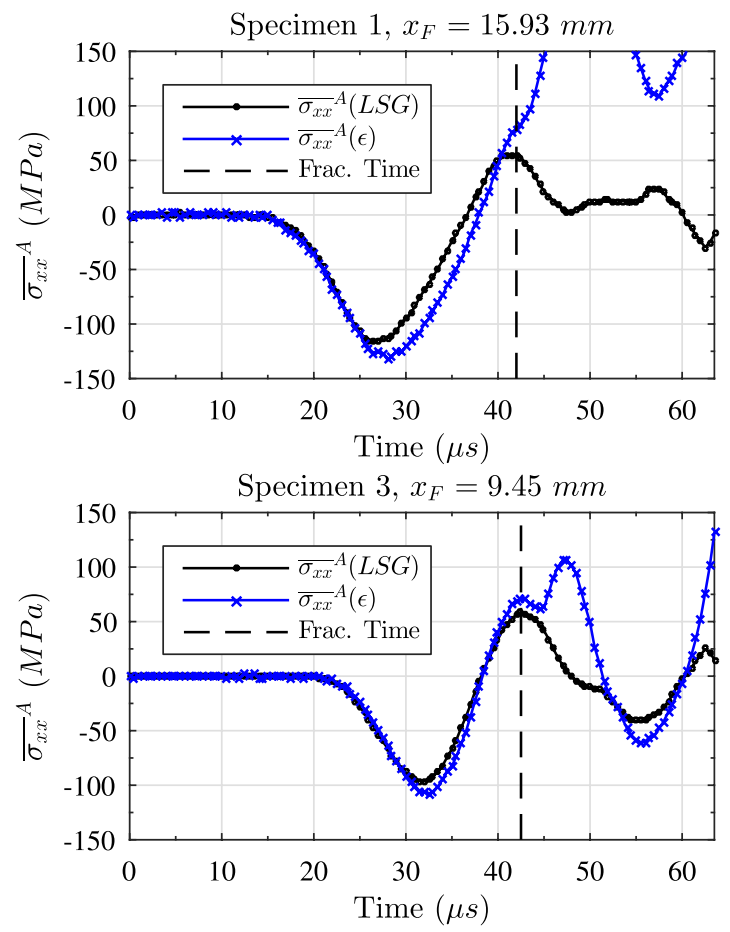

Fig. 25 Comparison of the average stress in the gauge area calculated using the acceleration ${\overline{\sigma_{x x}}}^{A}(L S G)$ and the strains ${\overline{\sigma_{x x}}}^{A}(\varepsilon)$ for all remaining experimental specimens. The fracture time for each speci-

Table 8 Peak mean tensile (tens.) and compressive (comp.) strain rate over the virtual gauge area $\left(\bar{\varepsilon}_{x x}^{A}\right)$ at the fracture location for each experimental specimen

\begin{tabular}{lll}
\hline Specimen & $\begin{array}{l}\text { Peak tens. } \\
{\overline{\varepsilon_{x x}}}^{A}\left(\mathrm{~s}^{-1}\right)\end{array}$ & \begin{tabular}{l}
${\overline{\varepsilon_{x x}^{\prime}}}^{A}\left(\mathrm{~s}^{-1}\right)$ \\
\hline 1
\end{tabular} \\
\hline 2700 & -1900 \\
3 & 2900 & -1800 \\
4 & 3300 & -1800 \\
5 & 2000 & -1200 \\
6 & 2900 & -1800 \\
\hline
\end{tabular}

The limitation of through-thickness heterogeneity is discussed further in the "Future Work and Limitations" section.

In order to assess the strain rate associated with the measured strength of each specimen, the peak average strain rate over the virtual gauge area was extracted. The peak tensile and compressive average strain rate is provided for each specimen in Table 8 . The strain rate history for the virtual gauge area on specimen 4 is provided in Fig. 26. The peak strain rates observed at each fracture location are similar in magnitude to the maximum peak strain rates in Table 6 . All specimens experience a peak tensile strain rate on the order of $2000 \mathrm{~s}^{-1}$.
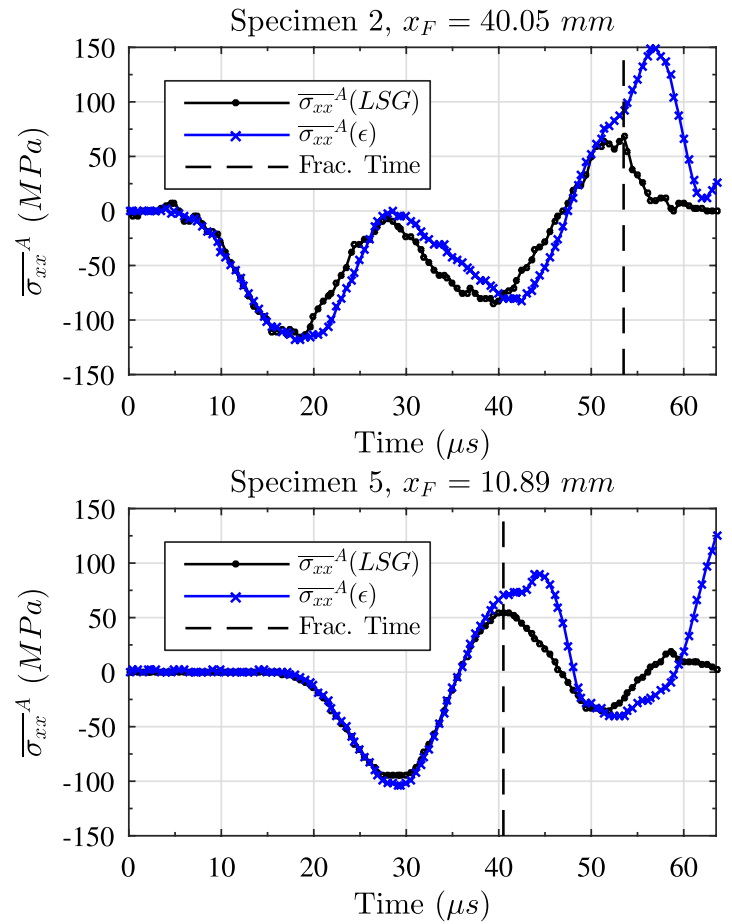

men is shown with a black dashed line and the axial location of fracture $x_{F}$ is given in the header of each sub-plot. Note that specimen 4 is shown in Fig. 24c

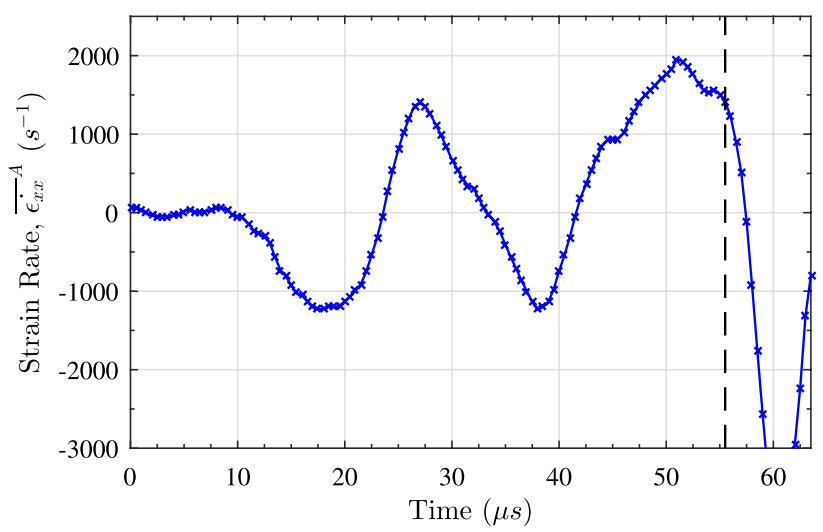

Fig. 26 Average strain rate history in the virtual gauge area (fracture location) for specimen 4

The results in Table 7 show that there is a significant increase in the strength when compared to the quasi-static value $(57 \%)$. This increase in strength is expected as the transverse properties of a unidirectional composite are dominated by the matrix material $[6,7]$. A previous study by Melin and Asp [1] showed a 37\% increase in the transverse tensile strength at a strain rate of $800 \mathrm{~s}^{-1}$. While a study by Gilat et al. [2] showed a 33\% increase in the transverse tensile strength at a strain rate of $400 \mathrm{~s}^{-1}$. These results contrast 
with the results of Kwon et al. which predict a $65 \%$ increase in the transverse tensile strength at a strain rate of $100 \mathrm{~s}^{-1}$. The results of the present study fall within the bounds of previous results. This variability in results with previous studies is likely to come from difference in polymer matrix chemistry, as previously mentioned.

The accuracy of the identified strength is dependent on a multitude of factors arising from the imaging system and the data processing method. The key parameters associated with the imaging system come from the spatial and temporal resolutions of the camera. A higher spatial resolution will allow for greater strain resolution and more precise identification of the fracture strain and location. A higher temporal resolution will allow for more accurate determination of the fracture time and fracture stress. For the present series of experiments, the frame rate was set to $2 \mathrm{MHz}$ such that a full reflection of the wave could be obtained. For all specimens in this study, it was observed that both measures of stress diverged significantly within a single multiple of the inter frame time. Thus, in future studies, it would be desirable to scale down the specimen such that a full reflection could be imaged while recording at a frame rate of $5 \mathrm{MHz}$. This would allow for greater temporal resolution for identifying the fracture time and allow for further temporal information to be obtained regarding the fracture processes in the material.

\section{Future Work and Limitations}

The results of this study have successfully demonstrated the feasibility of an IBII test for measuring the transverse properties of composites at high strain rates. However, there are a number of limitations that need to be discussed in order to better understand the scope of the results and the potential applications of the technique described here. These limitations are listed below.

\section{Through-Thickness Heterogeneity}

For all identification procedures used in this study, it is assumed that the stress and acceleration fields are uniform through the thickness. Any deviations from through-thickness uniformity will generate errors in the identified properties. Through-thickness dispersion was the suspected cause for the inconsistency between stress and strain for specimen 6 after the wave had reflected. This led to an inability to reliably identify the strength for this specimen. Future experiments need to be conducted using two cameras in a back-to-back configuration to assess the extent of any errors generated from through-thickness dispersion. However, as five of the six specimens tested did not exhibit this behaviour, it is unlikely that this is a significant limitation of this new test method.

\section{Stress Field Approximation}

This limitation refers to the use of the linear stress-gauge equation to approximate the stress at a particular location on the specimen for strength identification. Indeed, the linear stress gauge approximation is only appropriate if the actual stress distribution is linear across the specimen width at the considered cross-section. This was qualitatively verified for each specimen by comparing the stress calculated with the constitutive law with the stress calculated from the acceleration (see Fig. 25). Additionally, there are issues associated with temporal leakage effects in the acceleration. Any temporal leakage in the acceleration will be present in the derived stress information. The overall contribution of these temporal smearing effects is currently unknown but it is tractable using image deformation simulations.

With these limitations in mind, it is useful to consider the future development and potential applications of the IBII test presented in this work.

\section{Measurement Error Characterisation}

The use of image deformation simulations has been consistently mentioned as a method to assess the effects of camera noise, spatial and temporal resolutions. The accuracy of the identified properties are dependent on spatial and temporal resolutions of the camera, as well as the selected smoothing parameters. In order to fully characterise the errors arising from measurement resolution and noise, it is necessary to conduct image deformation simulations similar to refs. [31, 32]. Image deformation allows for the characterisation of random errors arising from noise as well as the systematic errors arising from the camera temporal and spatial resolutions. This will also allow for the effects of temporal leakage on the strength identification to be quantified. The authors are currently developing a software image deformation pipeline for this purpose. This image deformation pipeline will be coupled with explicit dynamics models that include cohesive surfaces to assess the strength identification procedure described previously.

\section{Strain Rate Control}

In this work, strain rate was not considered as part of the test design criteria. Obviously, it would be desirable to control the peak strain rate such that a wider range of strain rates could be tested. This will require further finite element simulations to understand which test configuration variables have most influence on the strain rate. It is expected that the speed and stiffness of the impactor are the two main drivers. 


\section{Application to Brittle Materials}

This test method can be applied to any material that has a compressive strength significantly higher than its tensile strength. Brittle materials that fall into this category include concrete, glass, graphite and ceramics. These materials are notoriously difficult to test at high strain rates for reasons that are similar to transverse testing of unidirectional composites (i.e. small strains to failure leading to corrupting inertial effects). The IBII test presented in this work has already been successfully applied to test the high strain rate properties of concrete in ref. [13], and has significant potential to provide new understanding of the high strain rate properties of other brittle materials.

\section{Shear/Tension and Through-Thickness Testing}

The present study focused on the in-plane transverse properties of a uni-directional composite. The current methodology can be modified to analyse coupled shear/tension testing by using samples cut at various angles to the fibre direction. Another extension of the present method would be to analyse the through-thickness tensile strength of thick composite plates using a scaled-down test sample.

In summary, the design space for the IBII test proposed in this study is significant. The ultra-high speed camera technology that is the foundation of this work will only improve in the future. Increases in both spatial and temporal resolutions of ultra-high speed cameras will lead to reduced measurement errors and increased efficacy of the test method proposed herein. It is anticipated that this technique could become a new standard test in the future.

\section{Conclusion}

This work presents a new methodology for testing the transverse properties of fibre composites at high strain rates. The type of test proposed is an image-based inertial impact (IBII) test. This test uses a short input compressive pulse which reflects and becomes tensile causing failure of the test sample. Throughout the test, ultra-high speed full-field measurements are taken and the concept of acceleration as a load-cell is used to reconstruct stress information in the specimen. The IBII test was successfully used to identify the transverse elastic modulus and tensile strength of a carbon fibre composite at peak strain rates on the order of $2000-3000 \mathrm{~s}^{-1}$. The main findings of this study can be summarised as follows.

1. Explicit dynamics simulations are a reliable tool for designing this IBII test giving suitable design predictions for the required test configuration.
2. It is possible to cause transverse tensile failure in a composite material with a relatively low impact speed, on the order of 30 to $35 \mathrm{~ms}^{-1}$.

3. Measurement quality with current ultra-high speed camera technology is sufficient to allow for both stiffness and strength identification in the same test.

4. The average transverse elastic modulus of the tested composite material was identified as $7.9 \mathrm{GPa}$ using the stress-gauge equation and $7.8 \mathrm{GPa}$ using the optimised virtual fields at a peak average strain rate on the order of $2000 \mathrm{~s}^{-1}$. This represents an $8 \%$ increase compared to the quasi-static value.

5. The average transverse tensile strength was identified as $58 \mathrm{MPa}$ at a strain rate on the order of $2000-3000 \mathrm{~s}^{-1}$. This represents a $57 \%$ increase compared to the quasistatic value.

Work is currently under way to extend the IBII method to combined shear-tension/compression testing as well as to the through-thickness properties of fibre composites. This new methodology can be applied to any brittle material that has a compressive strength greater than its tensile strength. Application of this new technique to brittle materials such as glass, concrete and ceramics will lead to experimental data that has previously not been available for these materials at high strain rates. Test methods based on full-field measurement have significant potential for high strain rate testing. Future advances in imaging technology will allow for the production of experimental data that was not previously attainable.

Acknowledgements This material is based on research sponsored by the Air Force Research Laboratory, under Agreement Number FA9550-17-1-0133. The authors are grateful to the grant programme manager, Dr David Garner from EOARD/AFOSR. The U.S. Government is authorized to reproduce and distribute reprints for Governmental purposes notwithstanding any copyright notation thereon. The views and conclusions contained herein are those of the authors and should not be interpreted as necessarily representing the official policies or endorsements, either expressed or implied, of the Air Force Research Laboratory or the U.S. Government. Dr Lloyd Fletcher and Prof. Fabrice Pierron acknowledge support from EPSRC through Grant EP/L026910/1.

\section{Compliance with Ethical Standards}

Data Provision All data supporting this study are openly available from the University of Southampton repository at: https://doi.org/10.5258/ SOTON/D0608.

Open Access This article is distributed under the terms of the Creative Commons Attribution 4.0 International License (http://creativeco mmons.org/licenses/by/4.0/), which permits unrestricted use, distribution, and reproduction in any medium, provided you give appropriate credit to the original author(s) and the source, provide a link to the Creative Commons license, and indicate if changes were made. 

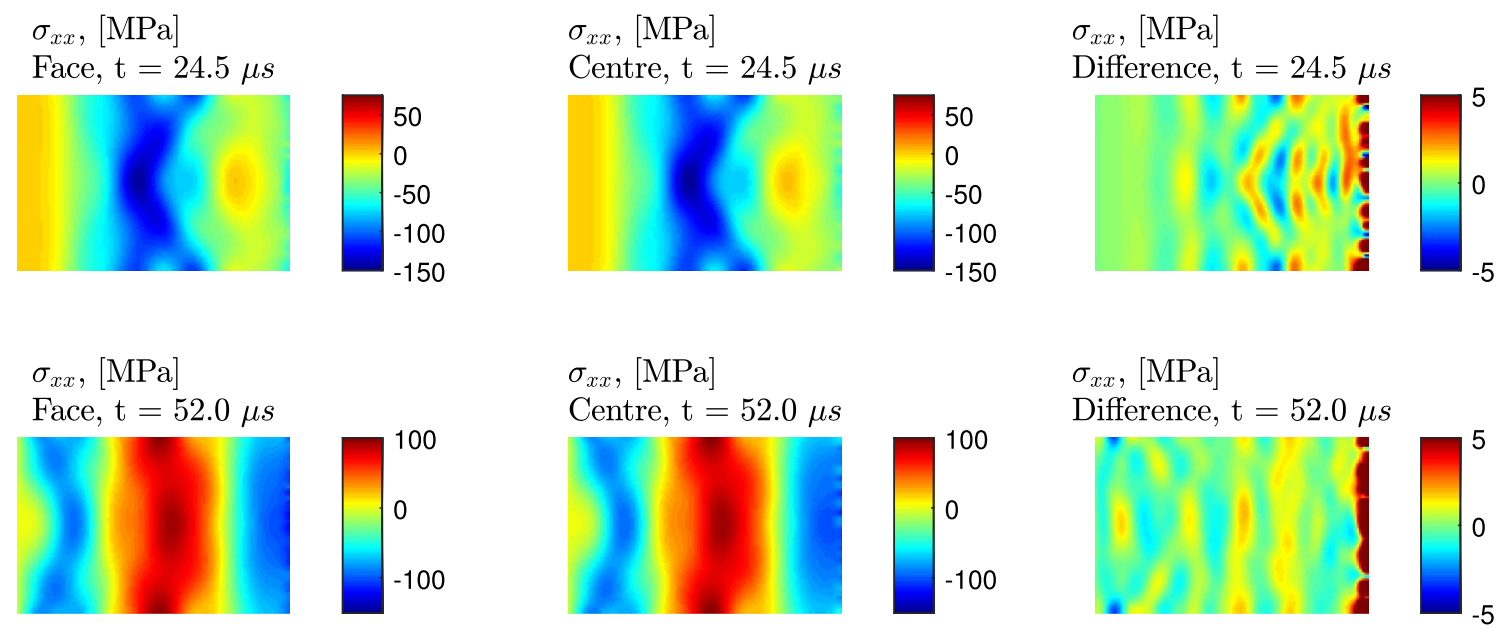

Fig. 27 Comparison of the in-plane stress component $\sigma_{x x}$ at the outer face and centre plane of the specimen for the 3D finite element model. The difference between the fields shows that there is minimal variation through-thickness for most of the surface of the specimen. (Color figure online) $\sigma_{z z},[\mathrm{MPa}]$

Face, $\mathrm{t}=24.5 \mu s$
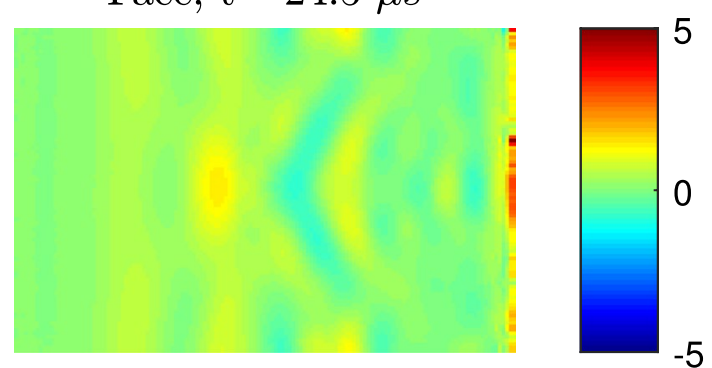

$\sigma_{z z},[\mathrm{MPa}]$

Face, $\mathrm{t}=52.0 \mu \mathrm{s}$
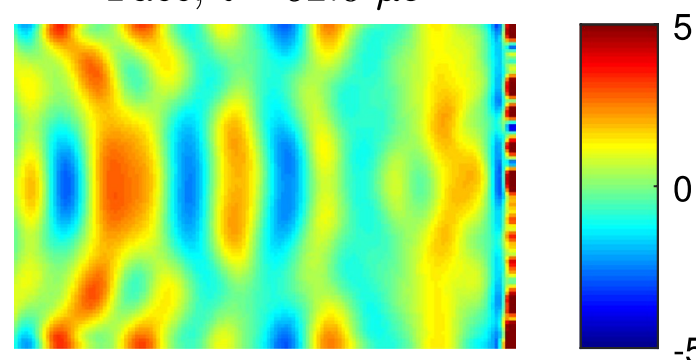

Fig. 28 Out of plane stress component $\sigma_{z z}$ for the outer face of the 3D finite element model. As the out of plane component is small compared to the in-plane stresses (see Fig. 27) the plane stress assumption is reasonably satisfied. (Color figure online)

\section{Appendix}

The purpose of this section is to verify the plane stress assumption and to assess the uniformity of the kinematic fields through-thickness using the 3D finite element model described in the "Finite Element Implementation" section. Here, the parameters of the finite element model were set to replicate the selected design case: waveguide length of $L_{w g}=50 \mathrm{~mm}$, projectile length of $L_{p}=25 \mathrm{~mm}$ and projectile velocity of at least $V_{p}=30 \mathrm{~m} / \mathrm{s}$.

The in-plane stress component $\sigma_{x x}$ was extracted for the outer face and central plane of specimen. A comparison of the stress fields at two different time points in the simulations is shown in Fig. 27. These time points were selected as the first corresponds to a point at which the loading pulse has mostly entered the specimen and the second corresponds to time point after the wave has reflected off the free edge. Analysing the difference of the two fields shows that it is small apart from near the impact edge. This difference likely arises from three dimensional effects where the sharp edge of the specimen is in contact with the flat surface of the waveguide. However, this difference quickly disappears within a few elements of the contact surface.

The out of plane stress component $\sigma_{z z}$ was also extracted from the surface of the specimen. The out of plane stress for the surface of the specimen is shown in Fig. 28. Comparing Fig. 28 to Fig. 27 shows that the out of plane stress is generally much less than the in-plane stress. This demonstrates that the assumptions of plane stress and through-thickness uniformity are reasonable.

\section{References}

1. Melin LG, Asp LE (1999) Effects of strain rate on transverse tension properties of a carbon/epoxy composite: studied by moiré photography. Compos A Appl Sci Manuf 30(3):305-316 
2. Gilat Amos, Goldberg Robert K, Roberts Gary D (2002) Experimental study of strain-rate-dependent behavior of carbon/epoxy composite. Compos Sci Technol 62(10-11):1469-1476

3. Taniguchi Norihiko, Nishiwaki Tsuyoshi, Kawada Hiroyuki (2007) Tensile strength of unidirectional CFRP laminate under high strain rate. Adv Compo Mater 16(2):167-180

4. Daniel IM, Werner BT, Fenner JS (2011) Strain-rate-dependent failure criteria for composites. Compos Sci Technol 71(3):357-364

5. Koerber H, Xavier J, Camanho PP (2010) High strain rate characterisation of unidirectional carbon-epoxy IM7-8552 in transverse compression and in-plane shear using digital image correlation. Mech Mater 42(11):1004-1019

6. Gerlach Robert, Siviour Clive R, Petrinic Nik, Wiegand Jens (2008) Experimental characterisation and constitutive modelling of RTM-6 resin under impact loading. Polymer 49(11):2728-2737

7. Jordan Jennifer L, Foley Jason R, Siviour Clive R (2008) Mechanical properties of Epon 826/DEA epoxy. Mech Time-Depend Mater 12(3):249-272

8. Gama Bazle A, Lopatnikov Sergey L, Gillespie Jr, John W (2004) Hopkinson bar experimental technique: a critical review. Appl Mech Rev 57(4):223-250

9. Lopatnikov SL, Gama BA, Krauthouser K, Gillespie G (2004) Applicability of the classical analysis of experiments with split Hopkins pressure bar. Tech Phys Lett 30(2):102-105

10. Sutton MA, Gilat A, Seidt J, Rajan S, Kidane A (2018) Full field deformation measurements in Tensile Kolsky Bar experiments: studies and detailed analysis of the early time history. J Dyn Behav Mater 4(1):95-113

11. Moulart R, Pierron F, Hallett SR, Wisnom MR (2010) Full-field strain measurement and identification of composites moduli at high strain rate with the virtual fields method. Exp Mech 51(4):509-536

12. Pierron F, Zhu H, Siviour C (2014) Beyond Hopkinson's bar. Phil Trans R Soc A 372(2023):20130195

13. Pierron F, Forquin P (2012) Ultra-high-speed full-field deformation measurements on concrete spalling specimens and stiffness identification with the virtual fields method. Strain 48(5):388-405

14. Avril S, Bonnet M, Bretelle A-S, Grédiac M, Hild F, Lenny P, Latourte F, Lemosse D, Pagano S, Pagnacco E, Pierron F (2008) Overview of identification methods of mechanical parameters based on full-field measurements. Exp Mech 48(4):381

15. Pierron Fabrice, Grédiac Michel (2012) The virtual fields method: extracting constitutive mechanical parameters from full-field deformation measurements. Springer, New York

16. Yoon S-H, Winters M, Siviour CR (2016) High strain-rate tensile characterization of EPDM rubber using non-equilibrium loading and the virtual fields method. Exp Mech 56(1):25-35

17. Koohbor Behrad, Kidane Addis, Wei-Yang Lu, Sutton Michael A (2016) Investigation of the dynamic stress-strain response of compressible polymeric foam using a non-parametric analysis. Int J Impact Eng 91:170-182

18. Reu PL, Miller TJ (2008) The application of high-speed digital image correlation. J Strain Anal Eng Des 43(8):673-688

19. Zhu Haibin (March 2015) A novel methodology for high strain rate testing using full-field measurements and the virtual fields methods. PhD, University of Troyes, France
20. Avril S, Grédiac M, Pierron F (2004) Sensitivity of the virtual fields method to noisy data. Comput Mech 34(6):439-452

21. Avril S, Pierron F (2007) General framework for the identification of constitutive parameters from full-field measurements in linear elasticity. Int J Solids Struct 44(14):4978-5002

22. Zhu H, Pierron F (2016) Exploration of Saint-Venant's principle in inertial high strain rate testing of materials. Exp Mech 56(1):3-23

23. Hsiao HM, Daniel IM, Cordes RD (1999) Strain rate effects on the transverse compressive and shear behavior of unidirectional composites. J Compos Mater 33(17):1620-1642

24. International Organization for Standardization (2009) ISO $527-$ 5:2009 - Plastics - Determination of tensile properties - Part 5: Test conditions for unidirectional fibre-reinforced plastic composites. International Organization for Standardization, Geneva, Switzerland

25. Grédiac M, Blaysat B, Sur F (2017) A critical comparison of some metrological parameters characterizing local digital image correlation and grid method. Exp Mech 57(6):871-903

26. Grédiac M, Sur F, Blaysat B (2016) The grid method for in-plane displacement and strain measurement: a review and analysis. Strain 52(3):205-243

27. Piro J-L, Grédiac M (2004) Producing and transferring low-spatial-frequency grids for measuring displacement fields with moiré and grid methods. Exp Tech 28(4):23-26

28. Pierron Fabrice, Cheriguene Rachid, Forquin Pascal, Moulart Raphael, Rossi Marco, Sutton M A (2011) Performances and limitations of three ultra high-speed imaging cameras for fullfield deformation measurements. Appl Mech Mater 70:81-86

29. Rossi Marco, Pierron Fabrice, Forquin Pascal (2014) Assessment of the metrological performance of an in situ storage image sensor ultra-high speed camera for full-field deformation measurements. Meas Sci Technol 25(2):025401

30. Herráez Miguel Arevallilo, Burton David R, Lalor Michael J, Gdeisat Munther A (2002) Fast two-dimensional phase-unwrapping algorithm based on sorting by reliability following a noncontinuous path. Appl Opt 41(35):7437-7444

31. Rossi Marco, Pierron Fabrice (2012) On the use of simulated experiments in designing tests for material characterization from full-field measurements. Int J Solids Struct 49(3):420-435

32. Lukić Bratislav, Saletti Dominique, Forquin Pascal (2017) Use of simulated experiments for material characterization of brittle materials subjected to high strain rate dynamic tension. Phil Trans R Soc A 375(2085):20160168

33. Lava P, Cooreman S, Coppieters S, De Strycker M, Debruyne D (2009) Assessment of measuring errors in DIC using deformation fields generated by plastic FEA. Opt Lasers Eng 47(7):747-753

34. Lava P, Cooreman S, Debruyne D (2010) Study of systematic errors in strain fields obtained via DIC using heterogeneous deformation generated by plastic FEA. Opt Lasers Eng 48(4):457-468

35. Rossi M, Lava P, Pierron F, Debruyne D, Sasso M (2015) Effect of DIC spatial resolution, noise and interpolation error on identification results with the VFM. Strain 51(3):206-222

36. Kwon Junbeom, Choi Jaeyoung, Huh Hoon, Lee Jungju (2017) Evaluation of the effect of the strain rate on the tensile properties of carbon-epoxy composite laminates. J Compos Mater 51(22):3197-3210 